\title{
Plasmacytoid dendritic cells from mouse tumor-draining lymph nodes directly activate mature Tregs via indoleamine 2,3-dioxygenase
}

\author{
Madhav D. Sharma,, 1 Babak Baban, ${ }^{3}$ Phillip Chandler,,2,4 De-Yan Hou,,2 Nagendra Singh, ${ }^{2}$ \\ Hideo Yagita, ${ }^{5}$ Miyuki Azuma, ${ }^{6}$ Bruce R. Blazar,7 Andrew L. Mellor, ${ }^{2,4}$ and David H. Munn ${ }^{1,2}$

\begin{abstract}
${ }^{1}$ Department of Pediatrics, School of Medicine, ${ }^{2}$ Immunotherapy Center and Cancer Center, ${ }^{3}$ Department of Pathology, and ${ }^{4}$ Department of Medicine, School of Medicine, Medical College of Georgia, Augusta, Georgia, USA. ${ }^{5}$ Department of Immunology, Juntendo University School of Medicine, Bunkyo-ku, Tokyo, Japan. ${ }^{6}$ Department of Molecular Immunology, Tokyo Medical and Dental University, Bunkyo-ku,
\end{abstract} \\ Tokyo, Japan. 'Department of Pediatrics and Division of Blood and Marrow Transplantation, University of Minnesota, Minneapolis, Minnesota, USA.
}

\begin{abstract}
A small population of plasmacytoid DCs (pDCs) in mouse tumor-draining LNs can express the immunoregulatory enzyme indoleamine 2,3-dioxygenase (IDO). We show that these $\mathrm{IDO}^{+} \mathrm{pDCs}$ directly activate resting $\mathrm{CD}^{+} \mathrm{CD}^{2} 5^{+} \mathrm{Foxp}^{+}{ }^{+}$Tregs for potent suppressor activity. In vivo, Tregs isolated from tumor-draining $\mathrm{LNs}$ were constitutively activated and suppressed antigen-specific $\mathrm{T}$ cells immediately ex vivo. In vitro, $\mathrm{IDO}^{+} \mathrm{pDCs}$ from tumor-draining LNs rapidly activated resting Tregs from non-tumor-bearing hosts without the need for mitogen or exogenous anti-CD3 crosslinking. Treg activation by $\mathrm{IDO}^{+} \mathrm{pDCs}$ was $\mathrm{MHC}$ restricted, required an intact amino acid-responsive GCN2 pathway in the Tregs, and was prevented by CTLA4 blockade. Tregs activated by IDO markedly upregulated programmed cell death 1 ligand 1 (PD-L1) and PD-L2 expression on target DCs, and the ability of Tregs to suppress target $\mathrm{T}$ cell proliferation was abrogated by antibodies against the programmed cell death 1/PD-L (PD-1/PD-L) pathway. In contrast, Tregs activated by anti-CD3 crosslinking did not cause upregulation of PD-Ls, and suppression by these cells was unaffected by blocking the PD-1/PD-L pathway. Tregs isolated from tumor-draining LNs in vivo showed potent PD-1/PD-L-mediated suppression, which was selectively lost when tumors were grown in IDO-deficient hosts. We hypothesize that $\mathrm{IDO}^{+} \mathrm{pDCs}$ create a profoundly suppressive microenvironment within tumor-draining LNs via constitutive activation of Tregs.
\end{abstract}

\section{Introduction}

A subset of DCs in murine tumor-draining lymph nodes (TDLNs) can express high levels of the tryptophan-degrading enzyme indoleamine 2,3-dioxygenase (IDO) (1). In other settings, IDO has been shown to contribute to maternal tolerance toward the allogeneic fetus, regulation of autoimmune disorders, and creation of tolerance to transplanted tissues (2-4). Transfection of IDO into tumor cells protects them from immune-mediated rejection (5), while inhibiting IDO in tumor-bearing hosts allows conventional chemotherapy to disrupt tolerance toward established tumors and trigger anti-tumor immune responses $(6,7)$. Thus, IDO is emerging as a potentially important tolerogenic mechanism in patients with cancer (8).

In vitro studies of $\mathrm{IDO}^{+} \mathrm{DCs}$ from murine TDLNs have shown that these cells are potently and dominantly suppressive for $\mathrm{T}$ cell activation $(1,7,9)$. Even a small minority of $\mathrm{IDO}^{+} \mathrm{DCs}$ is capable of inhibiting all $\mathrm{T}$ cell responses in culture, including dominant inhibition of $\mathrm{T}$ cells responding to antigens presented by other nonsuppressive APCs (1). In vivo, pharmacologic activation of the

Nonstandard abbreviations used: $\mathrm{B} 6, \mathrm{C} 57 \mathrm{BL} / 6$; GCN2, general control nonderepressing-2 kinase; IDO, indoleamine 2,3-dioxygenase; 1MT, 1-methyl-D-tryptophan; OVA, chicken ovalbumin; PD-1, programmed cell death 1; PD-L, PD ligand; PDC, plasmacytoid DC; TDLN, tumor-draining LN.

Conflict of interest: D.H. Munn and A.L. Mellor have intellectual property interests in the therapeutic use of IDO and IDO inhibitors and receive consulting income from NewLink Genetics Inc.

Citation for this article: J. Clin. Invest. 117:2570-2582 (2007). doi:10.1172/JCI31911.
IDO pathway systemically can completely inhibit clonal expansion of large numbers of alloreactive T cells (10). However, the number of $\mathrm{IDO}^{+} \mathrm{DCs}$ in that become activated in spleen or TDLNs is tiny (less than $1 \%$ of total cells, and typically less than $25 \%$ of total DCs), and it is unclear how the effects of IDO could create such potent and dominant immunosuppression.

Recently it has been shown that IDO can bias naive $\mathrm{CD}^{+} \mathrm{T}$ cells to differentiate into Foxp $3^{+}$Tregs in vitro (11). This important finding thus linked IDO to the potent Treg system, which is known to be a key mechanism of immunosuppression in tumorbearing hosts (12). However, de novo differentiation of Tregs from naive precursor cells is a slow process, requiring many days, whereas we knew from in vitro studies that IDO created dominant suppression within hours (prior to the first cell division of the suppressed T cells) $(9,13)$. Therefore we hypothesized that there existed a pathway by which IDO could directly activate the latent suppressor function of mature, preexisting Tregs; and further, that this pathway would be active in TDLNs in vivo.

\section{Results}

Tregs from TDLNs are bighly activated. We first tested the activation status of Tregs from TDLNs. B16 melanoma tumor cell lines were implanted in syngeneic C57BL/6 (B6) mice. Cell lines included B78H1-GM-CSF (a subline of B16 transfected with GM-CSF; ref. 14), the noninfected B16F10 subline of B16, and B16-OVA (the B16F10 subline transfected with ovalbumin). Mice were studied on days 7-11 after tumor implantation. All TDLNs contained a 
A

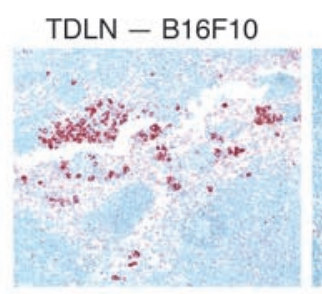

Contralateral LN

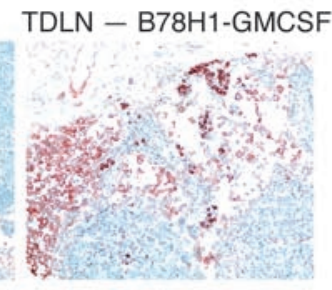

C

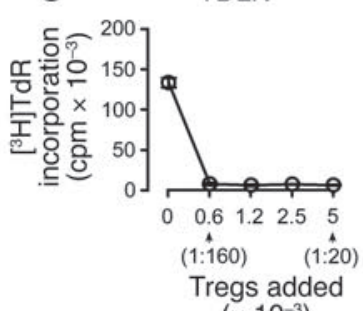

$\left(\times 10^{-3}\right)$
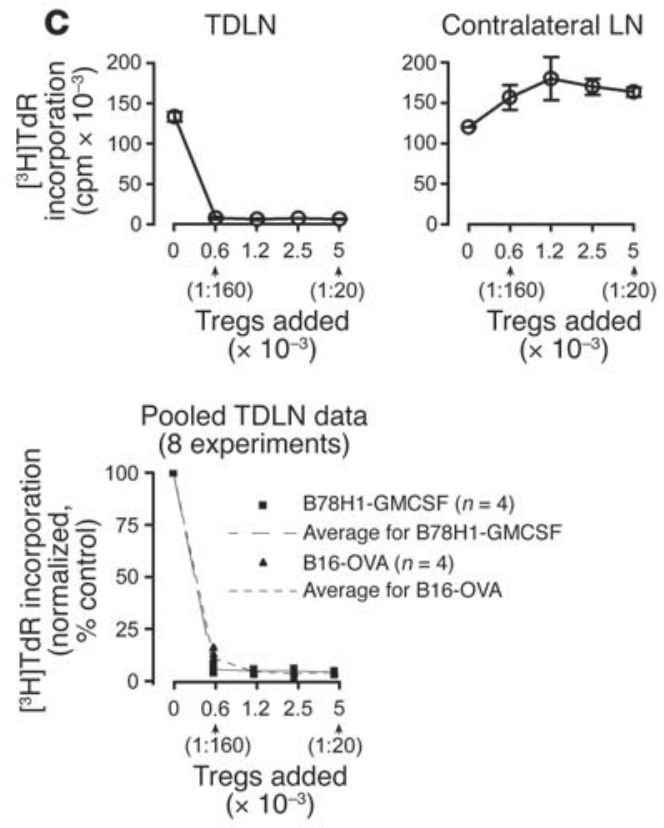

B

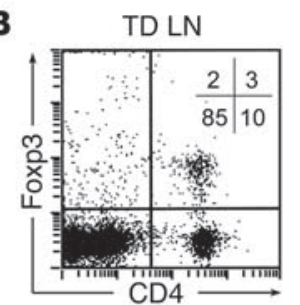

Contralateral LN

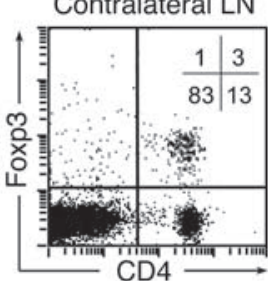

D

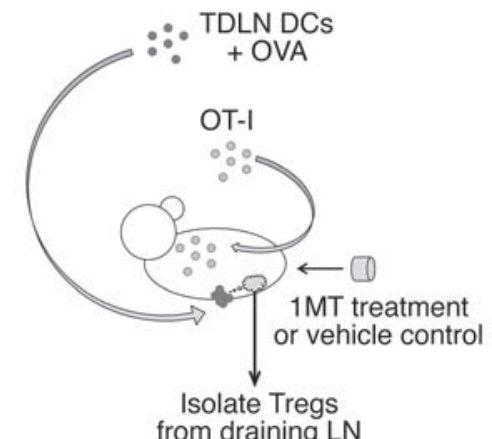

from draining $L N$

Ex vivo Treg supressor assay

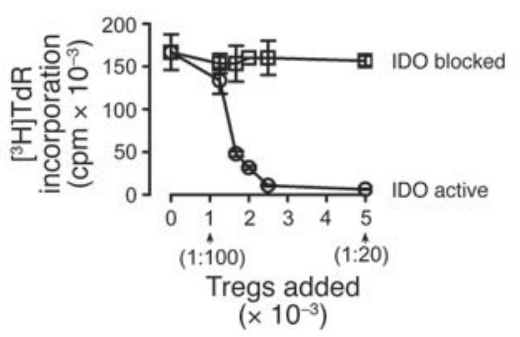

Figure 1

Treg activation by DCs from TDLNs. (A) Contralateral LNs and TDLNs from mice with B16F10 and B78H1-GM-CSF tumors (day 7-11). B16OVA tumors were identical to B16F10. Red color identifies IDO by immunohistochemistry. One representative of 3-6 experiments per cell line. Original magnification, $\times 200$. (B) TDLNs and contralateral LNs were stained for CD4 and intracellular Foxp3. Numbers indicate quadrant percentages. Representative of 6 experiments using B16-OVA and B78H1-GM-CSF. (C) Tregs (CD4+CD25+) from TDLNs and contralateral LNs were sorted and added to readout assays, which were comprised of $1 \times 10^{5} \mathrm{~A} 1 \mathrm{~T}$ cells plus CBA DCs plus $\mathrm{H}-\mathrm{Y}$ peptide. Proliferation (incorporation of $\left[{ }^{3} \mathrm{H}\right]$ thymidine deoxyribose, $\left.\left[{ }^{3} \mathrm{HTdR}\right]\right)$ is shown for a representative experiment. In all similar figures, the ratio of Tregs to A1 cells is shown below the axis (bars show SD of replicate wells). The lower graph shows data from 8 independent experiments using the tumor types shown (cpm were normalized to the proliferation in control assays receiving no Tregs, to permit comparison across experiments). (D) CD11 $\mathrm{c}^{+} \mathrm{DCs}$ were harvested from TDLNs, pulsed with OVA peptide, and injected subcutaneously into recipient mice preloaded with OT-I. One group of mice received implantable sustained-release 1MT pellets at $5 \mathrm{mg} /$ day (IDO blocked), while the other received vehicle control pellets (IDO active). After 4 days, the LNs draining the site of DC injection were harvested and the Tregs sorted and tested in vitro for spontaneous suppressor activity in readout assays (A1 T cells + CBA DCs). Representative of 3 experiments; bars show SD of replicate wells.

population of cells that constitutively expressed IDO (Figure 1A), which was not seen in non-TDLNs (contralateral LNs). We have previously shown (1) that these $\mathrm{IDO}^{+}$cells are a subset of DCs expressing plasmacytoid surface markers $\left(\mathrm{CD} 11 \mathrm{c}^{+} \mathrm{B} 220^{+}\right)$and coexpressing the marker CD19 (shown in Supplemental Figure 1; supplemental material available online with this article; doi:10.1172/ JCI31911DS1). The IDO ${ }^{+}$cells in TDLNs of all 3 tumor lines were similar; for most cell-sorting experiments B78H1-GM-CSF tumors were used, as in our previous publications $(1,9)$, because these gave the highest yield of pDCs (Supplemental Figure 1). However, pDCs from tumors without GM-CSF gave similar functional results, and all key findings were confirmed with both types of tumors.

Figure 1B shows analysis of Foxp $3^{+} \mathrm{CD} 4^{+}$Tregs in TDLNs. Both the TDLN and contralateral LNs contained a similar percentage of Tregs. However, when these Tregs were sorted by flow cytometry $\left(\mathrm{CD}^{+} \mathrm{CD} 25^{+}\right.$cells, more than $90 \%$ Foxp $\left.^{+}\right)$and tested for functional suppressor activity, the Tregs from TDLNs were potently and spontaneously suppressive, whereas the Tregs from contralateral LNs showed no spontaneous suppressor activity (Figure 1C). Tregs from TDLNs showed essentially complete suppression at a ratio of Tregs to readout $\mathrm{T}$ cells of greater than 1:100, which was as potent as the most highly activated Tregs achievable in vitro $(15,16)$. In these experiments, the goal was to test whether the Tregs from TDLNs were constitutively activated in vivo (as opposed to becoming activated during the readout assay). We therefore selected a readout system that was MHC mismatched to the B6 Tregs (comprised of TCR-transgenic A1 T cells and splenic DCs, both on the CBA strain background). The use of an allogeneic readout assay minimized any possible activation of the Tregs by the APCs in the readout assay, and no additional mitogen or 
A
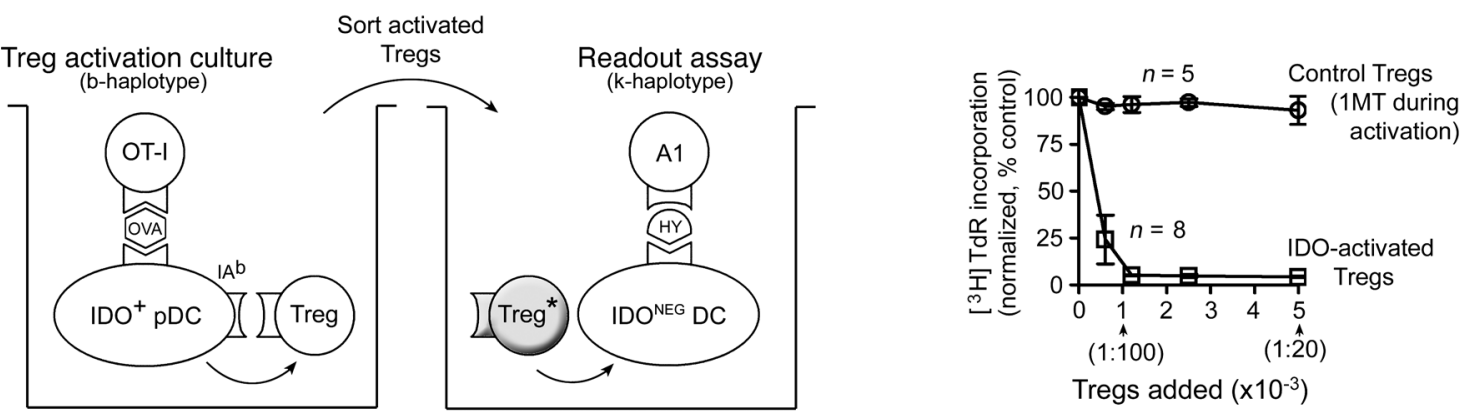

B

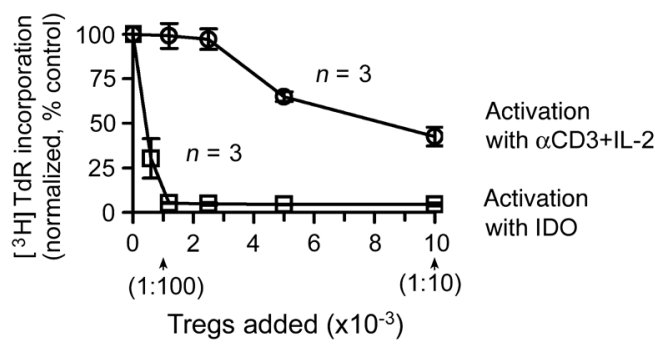

c

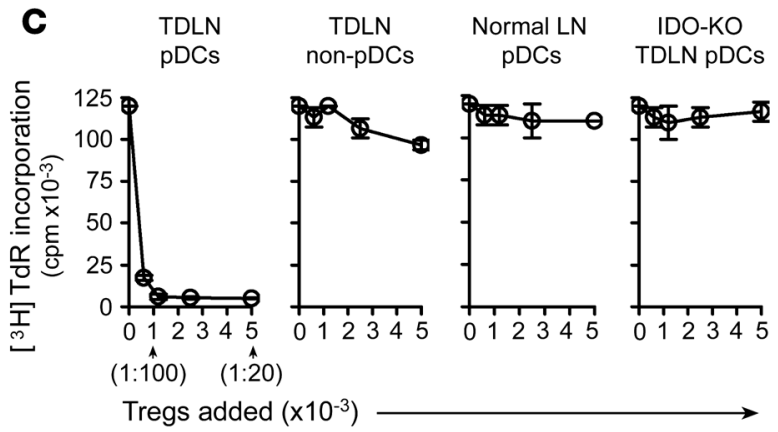

D

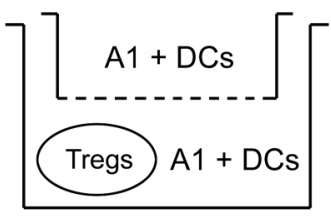

Control Tregs (1MT during activation)
IDO-activated Tregs

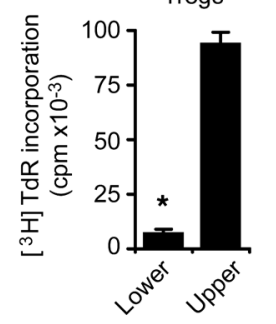

E

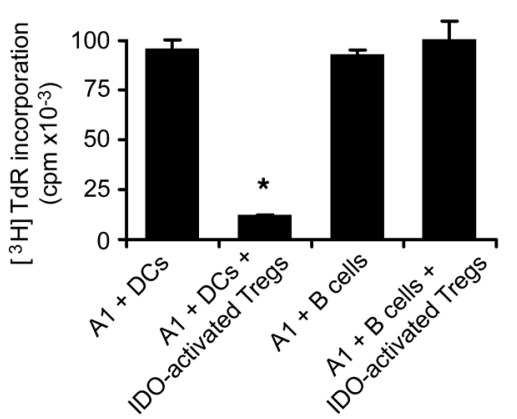

\section{Figure 2}

Activation of Tregs by IDO in vitro. (A) Resting Tregs were cocultured with TDLN pDCs plus OT-I plus feeder cells (all on B6 background, MHC b-haplotype) as described in Methods (IDO-activated Tregs). After 2 days the Tregs were re-sorted and added to readout assays (A1 T cells + CBA DCs, k-haplotype background). Controls Tregs were activated in identical cultures with $1 \mathrm{MT}$ added to block IDO activity. Graph shows the mean of 5-8 pooled experiments, using pDCs from B78H1-GM-CSF and B16-OVA tumors; error bars show SD. (B) Tregs were activated as described above or in identical cultures containing 1MT (to block IDO) and anti-CD3 mAb ( $\alpha$ CD3) plus IL-2 (IDO-activated Tregs). After 2 days, Tregs were re-sorted and tested in readout assays. Data points show the means for pooled values from 3 independent experiments. (C) Tregs were activated in cocultures as described above, and APCs were either TDLN pDCs, non-pDC fraction from the same TDLN (CD11 ${ }^{+}$B220-), pDCs from mice without tumors, or TDLN pDCs from IDO-KO mice. Graphs show 1 representative of 3-4 similar experiments for each group (bars show SD of replicate wells). (D) Tregs were activated with TDLN pDCs as described above, with or without 1MT. Tregs were re-sorted and added to readout assays in the lower chamber of transwell plates; upper chambers received readout assays without Tregs. Thymidine incorporation was measured separately in each chamber. One of 3 experiments; ${ }^{*} P<0.01$ by ANOVA. (E) IDO-activated Tregs were sorted and added to readout assays containing A1 T cells plus either CBA DCs or CBA B cells. One of 3 experiments; ${ }^{*} P<0.01$ by ANOVA.

anti-CD3 crosslinking was added. In assays of this type, resting Tregs do not show suppression $(17,18)$, whereas activated Tregs are suppressive (19). Thus, this assay allowed us to measure the degree of Treg activation in vivo.

$I D O^{+} D C s$ from TDLNs activate Tregs in vivo. To test the hypothesis that Treg activation in TDLNs might be related to the presence of IDO-expressing DCs, we isolated the DC population $\left(\mathrm{CD} 11 \mathrm{c}^{+}\right.$ cells) from TDLNs and transferred them to new, non-tumor-bearing hosts. (The phenotype of this mixed DC population has been previously described [ref. 1] and typically contained 30\%-50\% IDO-expressing $\mathrm{CD} 19^{+}$pDCs.) To test for the contribution of IDO, recipient mice were treated with the IDO-inhibitor drug
1-methyl-D-tryptophan (1MT) (7) beginning at the time of DC adoptive transfer or with vehicle control. Because we have shown that IDO does not become fully active until $\mathrm{IDO}^{+}$DCs present antigen to responding $\mathrm{T}$ cells (20), the DCs were pulsed with a peptide from chicken ovalbumin (OVA) and hosts pre-loaded with OVA-specific T cells (OT-I). Four days after DC transfer, host Tregs were sorted from LNs draining the site of DC injection and tested for suppressor activity. Figure 1D shows that Tregs exposed to DCs from TDLNs became potently activated and this activation was blocked when recipient mice were treated with $1 \mathrm{MT}$. Thus, the DC fraction from TDLNs, by itself, was sufficient to activate resting Tregs in new hosts, in an IDO-dependent fashion. 
A
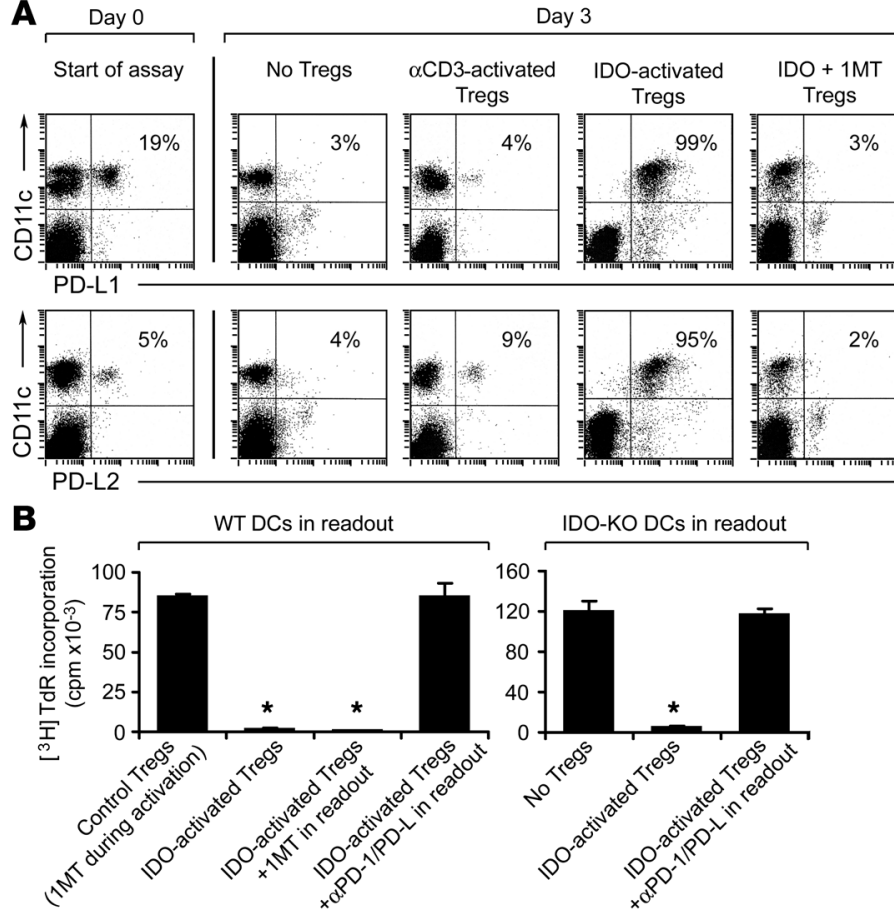

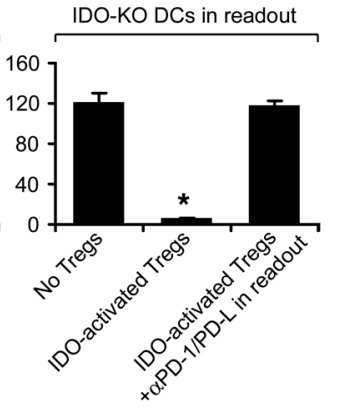

C
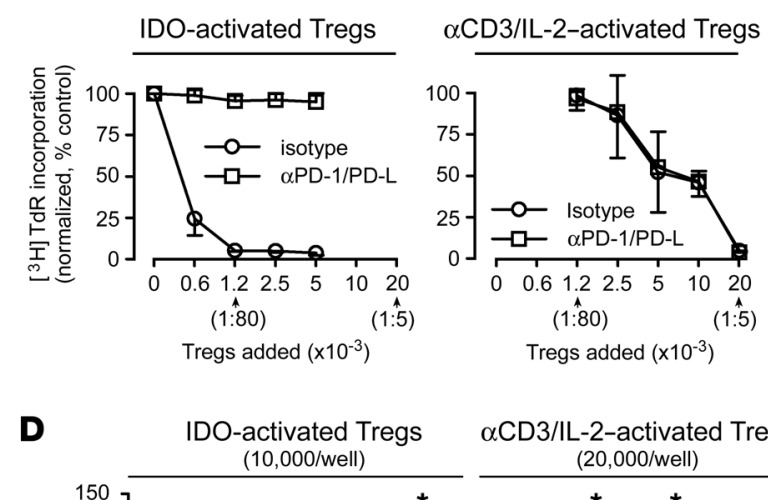

$\alpha$ CD3/IL-2-activated Tregs $(20,000 /$ well $)$

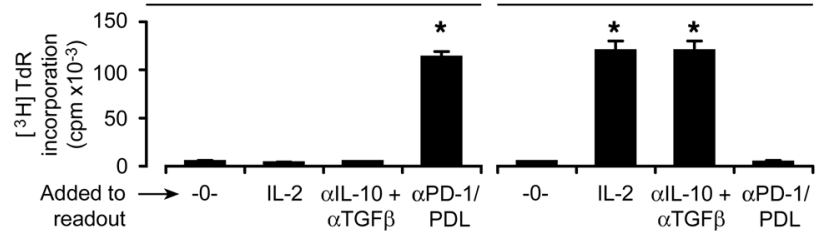

\section{Figure 3}

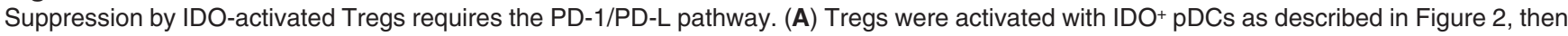
$1 \times 10^{4}$ sorted Tregs were added to readout assays (A1 T cells + CBA DCs). After 24 hours, cultures were harvested and stained for PD-L1 and $\mathrm{PD}$-L2 relative to $\mathrm{CD} 11 \mathrm{c}$. Percentages indicate the proportion of cells that are dual-positive (right-upper quadrant). One of 3 experiments. (B) IDO-activated Tregs (5,000/well) were added to readout assays (A1 T cells plus either wild-type CBA DCs or IDO-KO DCs on the CBA background). Readout assays received either no additive, $1 \mathrm{MT}$, or a cocktail of blocking antibodies against PD-1, PD-L1, and PD-L2 (50 $\mu \mathrm{g} / \mathrm{ml}$ each). Control Tregs received $1 \mathrm{MT}$ during the activation step. One of 3 experiments; ${ }^{\star} P<0.01$ by ANOVA. (C) Tregs were activated with IDO ${ }^{+}$pDCs or in identical cultures containing $1 \mathrm{MT}$ to block IDO and $\alpha \mathrm{CD} 3$ plus IL-2 to activate the Tregs. After sorting, Tregs were added to readout assays (A1 T cells + CBA DCs) with or without PD-1/PD-L-blocking antibodies as shown. Graphs show the mean \pm SD of 10 independent experiments with IDO-activated Tregs and 3 experiments with $\alpha$ CD3-activated Tregs, using TDLN pDCs from B78H1-GM-CSF and B16-OVA tumors. (D) IDO-activated Tregs $\left(1 \times 10^{4} /\right.$ well $)$ and $\alpha$ CD3/IL-2-activated Tregs $\left(2 \times 10^{4} /\right.$ well $)$ were prepared as described in the previous panel and added to readout assays with or without recombinant IL-2, anti-IL-10 plus anti-TGF- $\beta$ blocking antibodies (100 $\mu \mathrm{g} / \mathrm{ml}$ each), PD-1/PD-L-blocking antibodies, or no additive (-0-). Error bars show SD for replicate wells in 1 of 4 similar experiments. ${ }^{*}<0.01$ by ANOVA.

$I D O^{+}$pDCs from TDLNs activate resting Tregs in vitro. To study the mechanism of IDO-induced Treg activation, we used the 2-step model shown in Figure 2. Resting Tregs, from spleens of mice without tumors, were cocultured with $\mathrm{IDO}^{+}$DCs from TDLNs, then re-sorted and transferred to readout assays (A1 T cells + CBA DCs) to measure suppression. The IDO ${ }^{+}$DCs were enriched from TDLNs by sorting for the plasmacytoid DCs (pDCs) fraction, which we have previously shown to include essentially all of the $\mathrm{IDO}^{+} \mathrm{DCs}$ in TDLNs in our system (Supplemental Figure 1). Similar to human DCs (20), DCs from TDLNs required triggering signals from T cells at the time of antigen presentation in order to express functional IDO enzymatic activity. This was supplied by allowing the pDCs to present OVA peptide to OT-I. Cocultures also contained a feeder layer of T-depleted spleen cells, as described in Methods. After 30-48 hours, cocultures were harvested and the Tregs recovered by sorting for $\mathrm{CD}^{+}$cells (since the Tregs were the only $\mathrm{CD} 4^{+}$cells in the cocultures, they could be unambiguously recovered).

Figure $2 \mathrm{~A}$ shows that resting Tregs exposed to $\mathrm{IDO}^{+} \mathrm{pDCs}$ mediated potent suppression of $\mathrm{T}$ cell proliferation in readout assays. In contrast, if IDO was blocked by adding $1 \mathrm{MT}$ to the activation cultures, then the re-sorted Tregs showed no suppressor activity (similar to the resting Tregs from contralateral LNs shown in
Figure 1C). In the remainder of this article, we will refer to Tregs activated by $\mathrm{IDO}^{+}$pDCs from TDLNs as "IDO-activated Tregs," since IDO was necessary for activation, recognizing that additional signals besides IDO may also be supplied by these TDLN pDCs.

IDO-activated Tregs were able to suppress $\mathrm{CD}^{+} \mathrm{T}$ cells as well as $\mathrm{CD} 4^{+} \mathrm{T}$ cells in the readout assays (Supplemental Figure 2). Activation occurred within 30 hours and was sufficiently rapid that IDO-activated Tregs were able to suppress all proliferation of readout cells, even if the A1 cells and CBA DCs were added directly to the Treg activation assay at the beginning of cultures and allowed to activate in parallel (shown in Supplemental Figure 3). The A1 T cells in the readout assays were suppressed by activated Tregs, but they were not killed, as shown by the fact that recovery of $\mathrm{CD}^{+}$cells at the end of 3 days was $95 \% \pm 8 \%$ of the expected cell number compared with controls ( $n=5$ experiments), and Annexin V staining at the end of the 3-day assay was negative (Supplemental Figure 4).

Figure $2 \mathrm{~B}$ shows a quantitative comparison of IDO-activated Tregs versus the same Tregs activated using the widely used approach of anti-CD3 crosslinking (21). Both activation cultures contained identical cell populations, but the anti-CD 3 cultures received $1 \mathrm{MT}$ to block IDO plus anti-CD3 and recombinant IL-2 to 
A

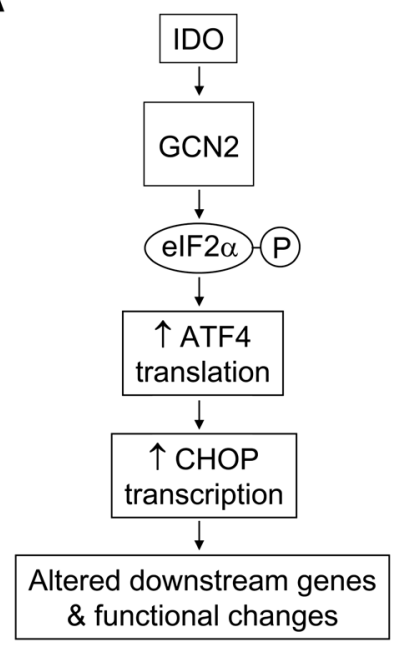

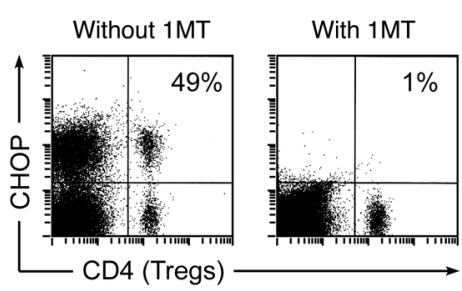

B

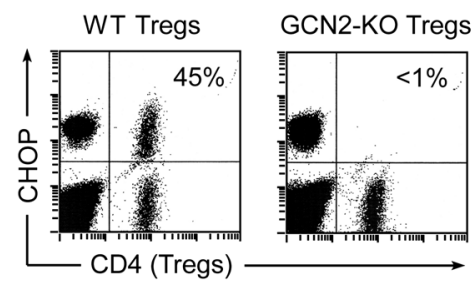

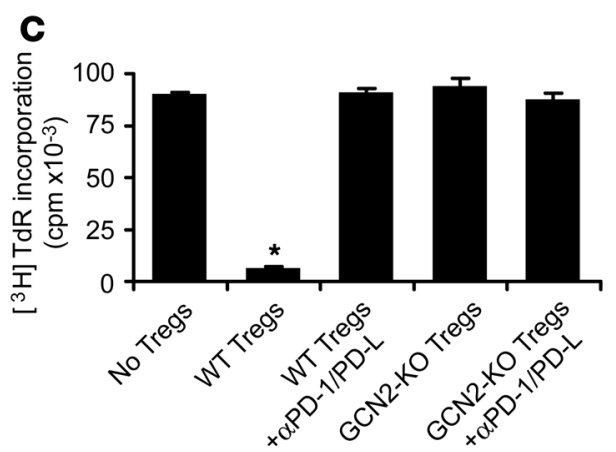

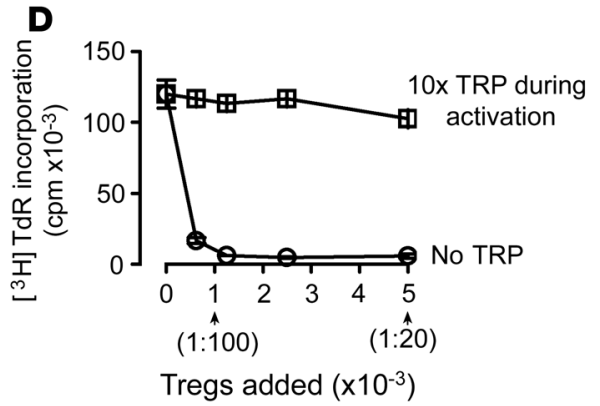

Figure 4

IDO-induced activation requires GCN2 in Tregs. (A) Activation cultures were set up with Tregs, TDLN pDCs, OT-I, and feeder cells, with or without $1 \mathrm{MT}$. After 2 days, intracellular staining was performed for $\mathrm{CHOP}$ expression in Tregs (CD4+ cells). The percentages show the fraction of Tregs that were $\mathrm{CHOP}^{+}$. One of 9 similar experiments. (B) As in the preceding panel, Tregs derived from wild-type mice are compared with GCN2-KO mice (each assay with OVA, without $1 \mathrm{MT}$ ). One of 3 experiments. (C) Tregs from GCN2-KO mice or wild-type controls were activated with IDO+ pDCs as $^{-}$ described in Figure 2 and re-sorted, and 5,000 Tregs were added to readout assays (A1 T cells + CBA DCs), with and without PD-1/PD-L-blocking antibodies. One of 3 similar experiments. ${ }^{*} P<0.01$ by ANOVA. (D) Tregs from wild-type mice were activated with IDO ${ }^{+}$pDCs, re-sorted, and tested in readout assays with and without added 10x tryptophan $(250 \mu \mathrm{M})$. Bars show SD for replicate wells. One of 3 similar experiments.

activate the Tregs. After activation and sorting, the IDO-activated Tregs mediated potent suppression, while the anti-CD3-activated Tregs were activated but quantitatively less suppressive (50\% inhibition at a Treg/target cell ratio of 1:10, which is consistent with the findings of others using the anti-CD3 system; refs. 16, 21).

Figure 2C shows similar cocultures, but with the TDLN pDCs replaced by various DCs that do not express IDO. The left graph (positive control) shows Tregs cocultured with TDLN pDCs $\left(\mathrm{IDO}^{+}\right)$. The middle left graph shows cocultures using the nonplasmacytoid (CD11 $\left.\mathrm{c}^{+} \mathrm{B} 220^{\mathrm{NEG}}\right) \mathrm{DCs}$ from the same TDLNs. The middle right graph shows cocultures using $\mathrm{pDCs}$ from LNs of mice without tumors. The right graph shows cultures containing $\mathrm{pDCs}$ isolated from TDLNs of tumors grown in IDO-knockout (IDO-KO) hosts. Only the plasmacytoid DC fraction derived from TDLNs and with an intact host IDO gene were able to activate Tregs. These data, combined with the complete abrogation of activation by $1 \mathrm{MT}$ (Figure $2 \mathrm{~A}$ ), support a mechanistic role for IDO in mediating Treg activation.

We next tested whether IDO-activated Tregs required physical contact with readout $\mathrm{T}$ cells in order to cause suppression (Figure 2D). IDO-activated Tregs were added to the lower well of transwell chambers, and readout cells (A1 T cells + CBA DCs) were placed in both the lower chamber (in contact with the Tregs) and the upper chamber (separated by a microporous membrane). Separate thymidine incorporation assays were performed on each chamber and showed that the IDO-activated Tregs suppressed those T cells with which they were in contact but had no effect on T cells separated across the membrane.
Suppression by IDO-activated Tregs requires the PD-1/PD-ligand pathway. Certain forms of $\mathrm{T}$ cell suppression by Tregs can be mediated indirectly via an effect on the target APCs (22). We therefore asked whether suppression by IDO-activated Tregs required the participation of the DCs in the readout assays. Figure 2E shows that IDOactivated Tregs were unable to suppress proliferation of A1 T cells when B cells were substituted instead of DCs as APCs used in the readout assay. Similar loss of suppression was seen when anti-CD3/ CD28-coated beads were substituted for the DCs (data not shown). This suggested that the suppressive effect of IDO-activated Tregs might be mediated indirectly via an effect on the target DCs.

One mechanism by which DCs may suppress T cells is the inhibitory programmed cell death 1 /programmed cell death 1 ligand (PD-1/PD-L) pathway $(23,24)$. While this pathway has not previously been described as a mediator of Treg suppression, related B7 family members have been linked to Treg-induced suppression (25). Figure 3A shows that IDO-activated Tregs caused upregulation of

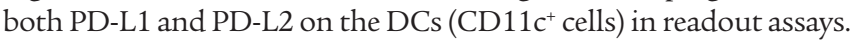
In contrast, PD-L expression by DCs was low in readout assays without Tregs and in readout assays receiving Tregs from activation cultures in which IDO was blocked with 1MT (Figure 3A). Even readout assays receiving Tregs that had been activated with anti-CD3 plus IL-2 did not show upregulation of PD-Ls on DCs (Figure 3A). Thus, the upregulation of PD-Ls on DCs appeared associated specifically with the form of Treg activation created by IDO.

We therefore asked whether blocking the PD-1/PD-L pathway in the readout assay would prevent suppression by IDO-activated Tregs. To ensure that the pathway was fully blocked, we added 
A

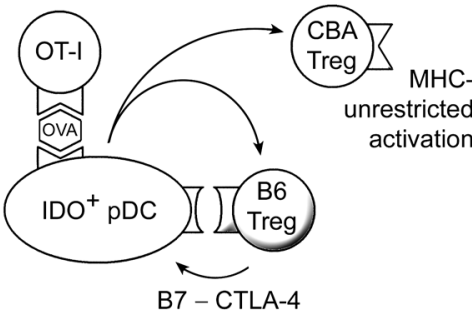

B7 - CTLA-4
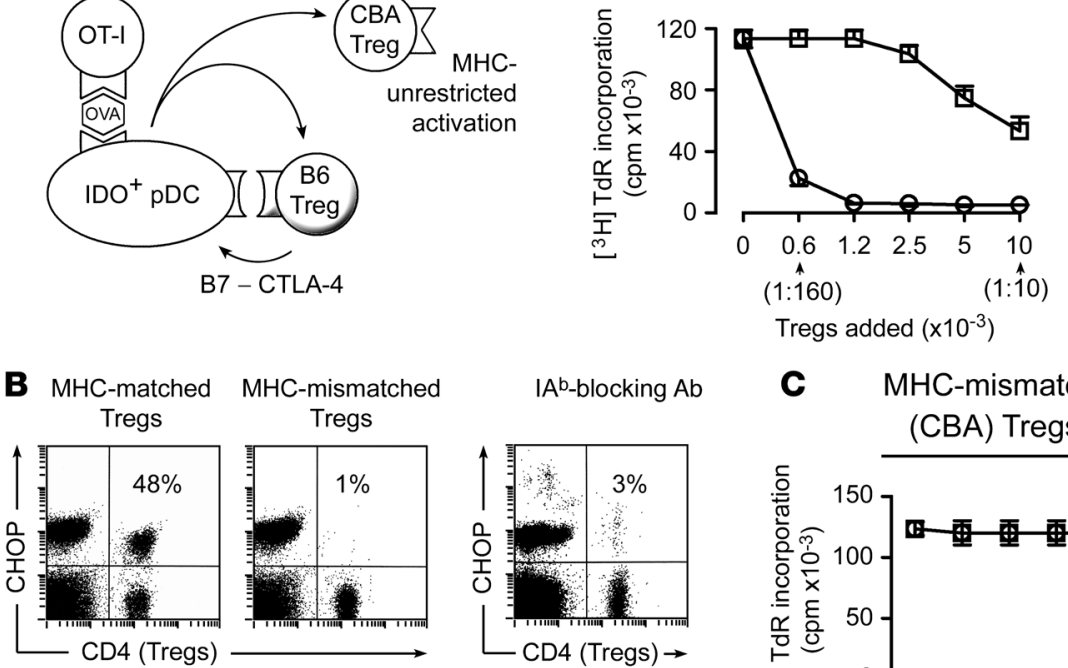

$\mathrm{A}^{\mathrm{b}}$-blocking $\mathrm{Ab}$

C

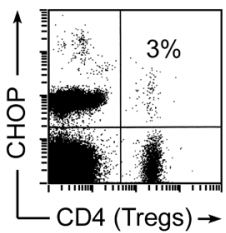

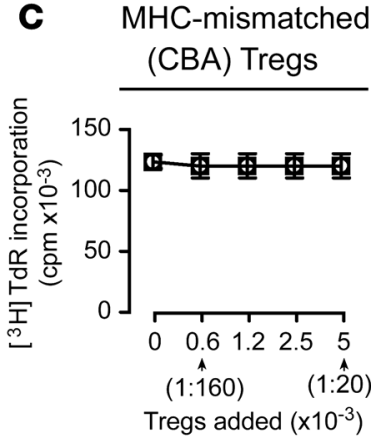

\section{Activation conditions}
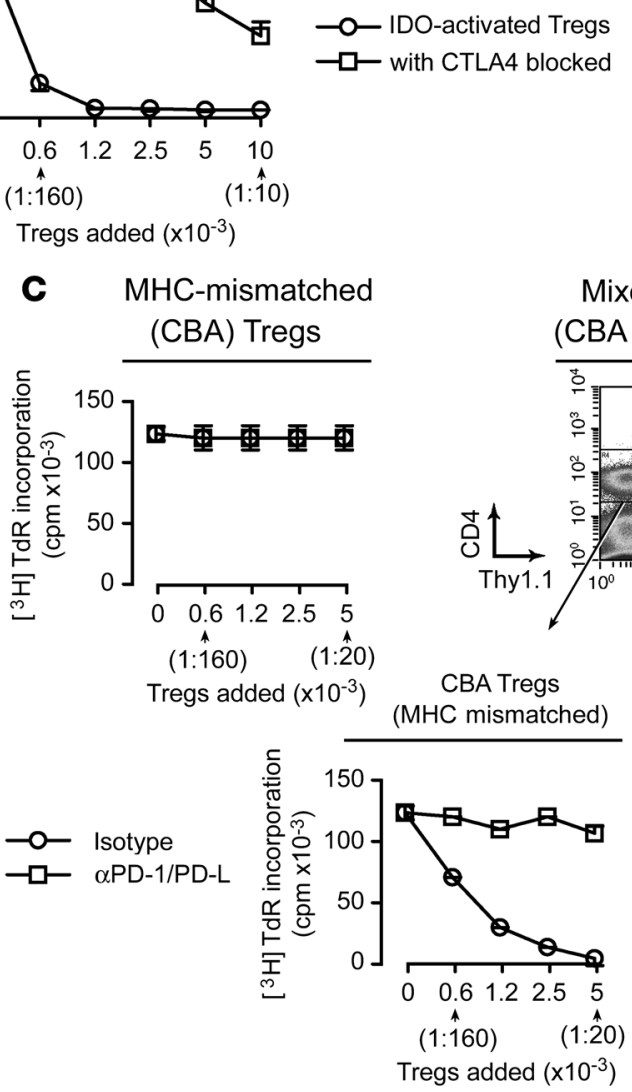

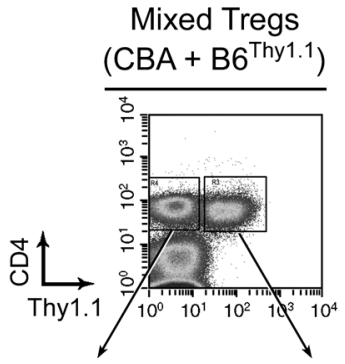

CBA Tregs

B6 Thy1.1 Tregs (MHC matched)

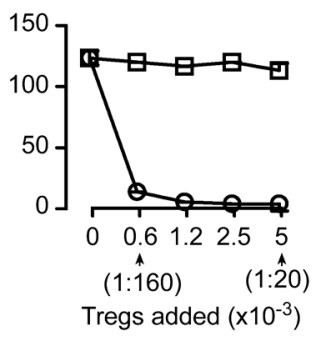

Figure 5

MHC-dependent and MHC-independent steps in IDO-induced Treg activation. (A) B6 Tregs were activated with IDO+ pDCs as described in Figure 2, with or without anti-CTLA4-blocking $\mathrm{mAb}(10 \mathrm{ug} / \mathrm{ml})$ during the activation step. Activated Tregs were re-sorted and tested in readout assays (A1 T cells + CBA DCs). Bars show SD for replicate wells in 1 of 4 similar experiments. (B) CHOP induction in Tregs is MHC restricted. Cultures were set up as described in Figure 4A and cells stained for CHOP after 2 days. The left plot shows assays using Tregs that were MHC matched to the $\mathrm{IDO}^{+}$pDCs (B6 background); the middle plot shows assays with MHC-mismatched (CBA) Tregs. The right plot shows cultures with MHC-matched B6 Tregs but with $100 \mu \mathrm{g} / \mathrm{ml}$ blocking antibody to $\mathrm{IA}^{\mathrm{b}}$. Controls without blocking antibody or with irrelevant antibody were similar to the first plot and are not shown. One of 4 experiments. (C) Left: Activation cocultures were set up as described in Figure 2 using MHC-mismatched (CBA) Tregs. After 2 days, CBA Tregs were re-sorted and added to readout assays (A1 T cells + CBA DCs). Right: Identical assays, except that CBA Tregs were mixed with Thy1.1 congenic B6 Tregs (10,000 each) during the activation cocultures, then each Treg population was re-sorted and tested separately. Error bars show SD for replicate wells in 1 of 3 similar experiments, using TDLN pDCs from B78H1-GM-CSF and B16-OVA tumors.

a cocktail of antibodies against PD-1, PD-L1, and PD-L2 to the readout assays. Blocking the $\mathrm{PD}-1 / \mathrm{PD}-\mathrm{L}$ pathway completely abrogated the ability of IDO-activated Tregs to suppress $\mathrm{T}$ cell proliferation (Figure 3B). In contrast, adding $1 \mathrm{MT}$ to the readout assay or using DCs from IDO-KO mice had no effect on T cell suppression. Thus, while IDO was strictly required to activate the Tregs initially (see Figure 2A), suppression of target cells by IDO-activated Tregs was independent of IDO and was dependent on the PD-1/PD-L pathway.

Figure 3C compares the role of the PD-1/PD-L pathway in suppression mediated by IDO-activated Tregs versus the suppression mediated by Tregs activated by anti-CD3 plus IL-2. Suppression by IDOactivated Tregs was completely prevented by blocking PD-1/PD-L in the readout assay, whereas suppression by anti-CD3-activated Tregs was unaffected by PD-1/PD-L blockade. In contrast, Figure 3D shows that suppression by anti-CD3-activated Tregs was fully reversed by adding recombinant IL-2 to the readout assay or by blocking IL-10 and TGF- $\beta$, although these manipulations had no effect on suppression by IDO-activated Tregs. Thus, the mechanisms of suppression by IDO-activated Tregs and anti-CD3-activated Tregs were distinct and could be unambiguously distinguished based on sensitivity to PD-1/PD-L blockade, exogenous IL-2, and IL-10/TGF- $\beta$ blockade.

GCN2 is required for Treg activation. We next asked whether Tregs responded to IDO via the GCN2 pathway. GCN2 is activated by reduced levels of amino acids, as might occur when IDO depletes tryptophan (26). We have previously shown that IDO activates GCN2 in $\mathrm{CD}^{+}$effector T cells, leading to cell-cycle arrest and anergy in these cells (9). As diagrammed in Figure 4, activation of GCN2 can be detected by measuring the downstream marker gene $\mathrm{CHOP} /$ gadd153 (9). Treg activation cultures were set up as described in the 
A

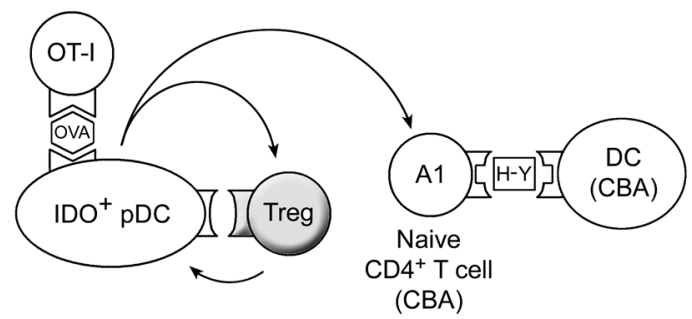

Gated CD4 ${ }^{+}$cells

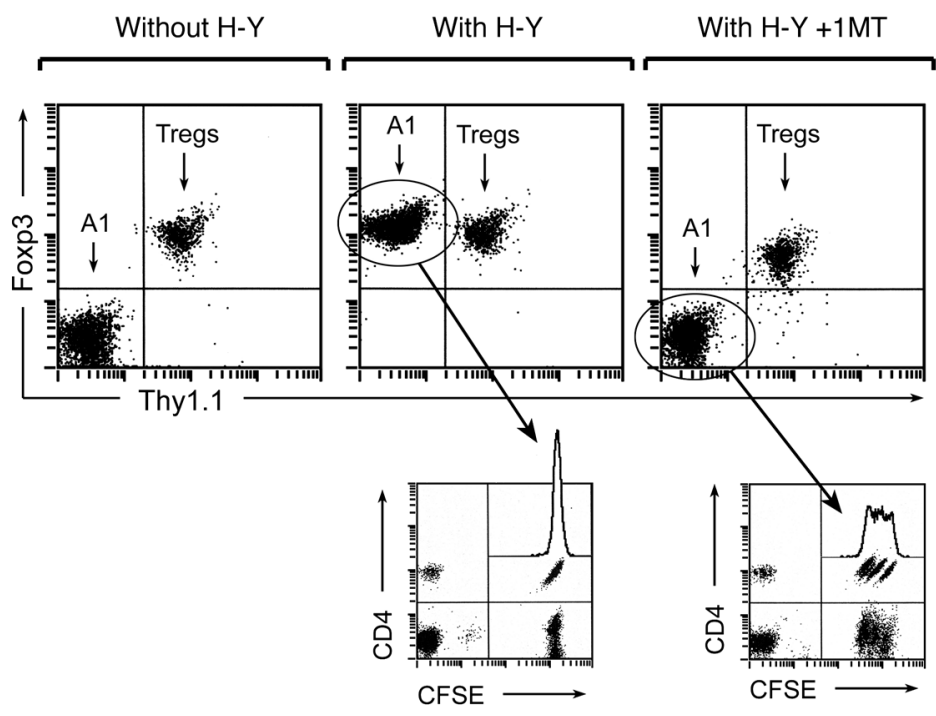

B

\author{
Activation together \\ in coculture
}

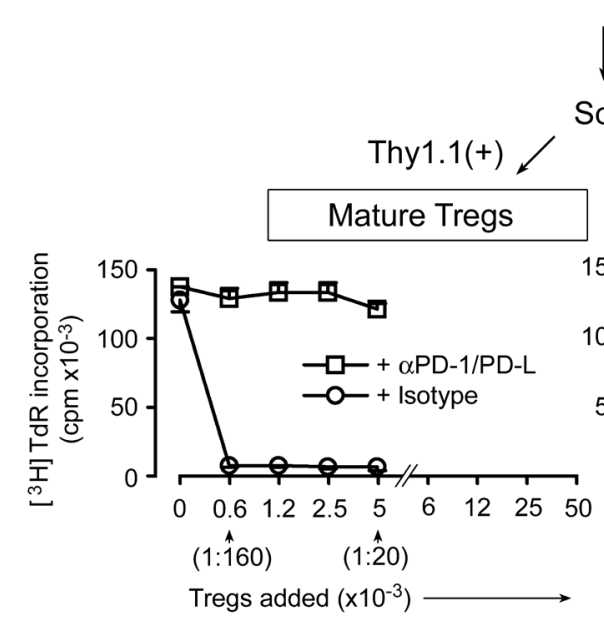

Figure 6

Direct activation of mature Tregs is more potent than de novo differentiation of new Tregs. (A) Activation cocultures were set up as described in Figure 2 using Thy1.1-congenic B6 Tregs. To these were added CD4+CD25- (naive, nonregulatory) T cells from A1 mice plus CBA spleen DCs. Parallel groups received either no $\mathrm{H}-\mathrm{Y}$ antigen for the $\mathrm{A} 1 \mathrm{cells}, \mathrm{H}-\mathrm{Y}$, or $\mathrm{H}-\mathrm{Y}$ plus $1 \mathrm{MT}$. (All cultures received OVA peptide for the OT-I). After 2 days, cocultures were stained for CD4, Foxp3, and Thy1.1. The smaller dot plots show similar cultures in which the A1 cells and OT-I were labeled with CFSE prior to addition, then analyzed for cell division at the end of the assay. CFSE histograms for the $\left.\mathrm{A}^{1} \mathrm{cells}^{\left(\mathrm{CD}^{+} \mathrm{CFSE}\right.}{ }^{+}\right)$are superimposed. One of 4 experiments. (B) Assays were set up as described in the previous panel, using Thy1.1 congenic Tregs plus nonregulatory $\mathrm{CD} 4{ }^{+} \mathrm{CD} 25^{-}$cells from wild-type $\mathrm{B} 6$ mice, activated with $\alpha \mathrm{CD} 3 \mathrm{mAb}$. Dot plots show upregulation of Foxp3 in this model using CD4 ${ }^{+} \mathrm{CD} 25^{-}$ cells prelabeled with CFSE. After 2 days the Treg and non-Treg populations were sorted separately based on Thy 1.1 expression and tested in readout assays ( $\mathrm{A} 1 \mathrm{~T}$ cells $+\mathrm{CBA} \mathrm{DCs})$. One of 3 similar experiments; error bars show SD.

Figure 2 legend and CHOP expression measured by intracellular staining after 2 days. Figure 4A shows that CHOP was upregulated when IDO was active and was expressed in both OT-I (visible as the CD4- population) and Tregs $\left(\mathrm{CD}^{+}\right)$. In these studies, approximately half of the Tregs upregulated CHOP, which could reflect an intrinsic heterogeneity in our $\mathrm{CD} 25^{+}$Treg population. Blocking IDO with $1 \mathrm{MT}$ abrogated CHOP expression in OT-I as expected and also prevented $\mathrm{CHOP}$ induction in Tregs, showing that both events were IDO dependent (Figure 4A). Figure 4B shows that Tregs derived from mice lacking functional GCN2 (GCN2-KO mice) showed no IDO-induced upregulation of CHOP. Consistent with this, GCN2-KO Tregs were unable to undergo functional activation by IDO (Figure 4C). GCN2-KO Tregs were still able to undergo anti-CD3-induced activation (data not shown), so they were not globally deficient in suppressor activity. Finally, Figure 4D shows that IDO-induced activation of wild-type Tregs was blocked by adding excess tryptophan to the activation cultures. Taken together, these data were consistent with the hypothesis that a tryptophan withdrawal stress, imposed by IDO and sensed via the GCN2 pathway, was required for Treg activation by $\mathrm{IDO}^{+} \mathrm{pDCs}$. 

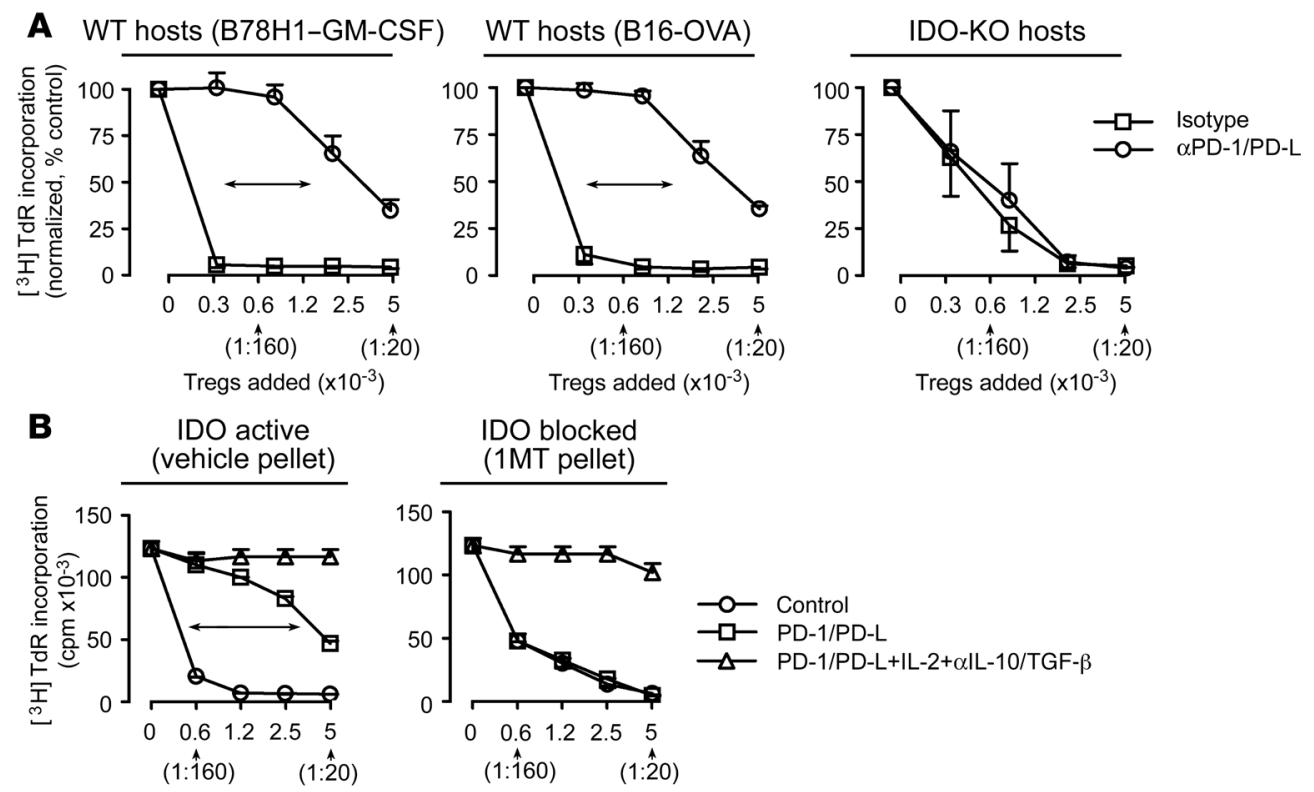

IDO blocked (1MT pellet)
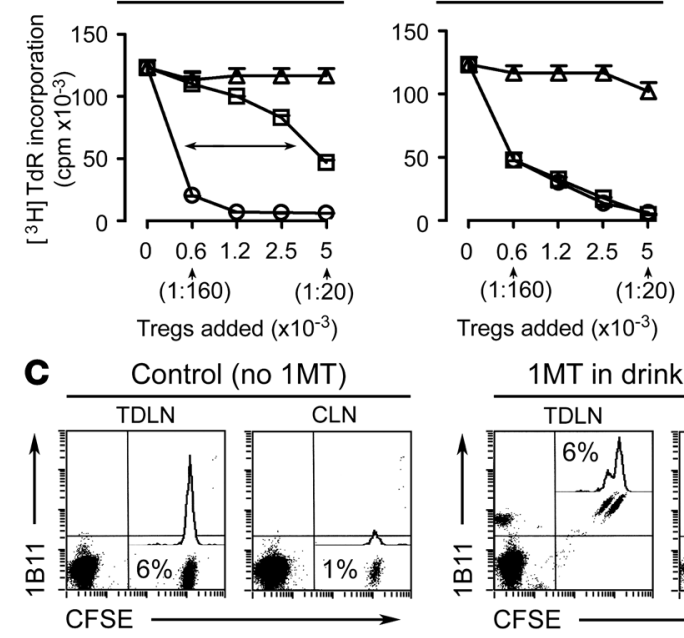

Tregs added $\left(\times 10^{-3}\right)$
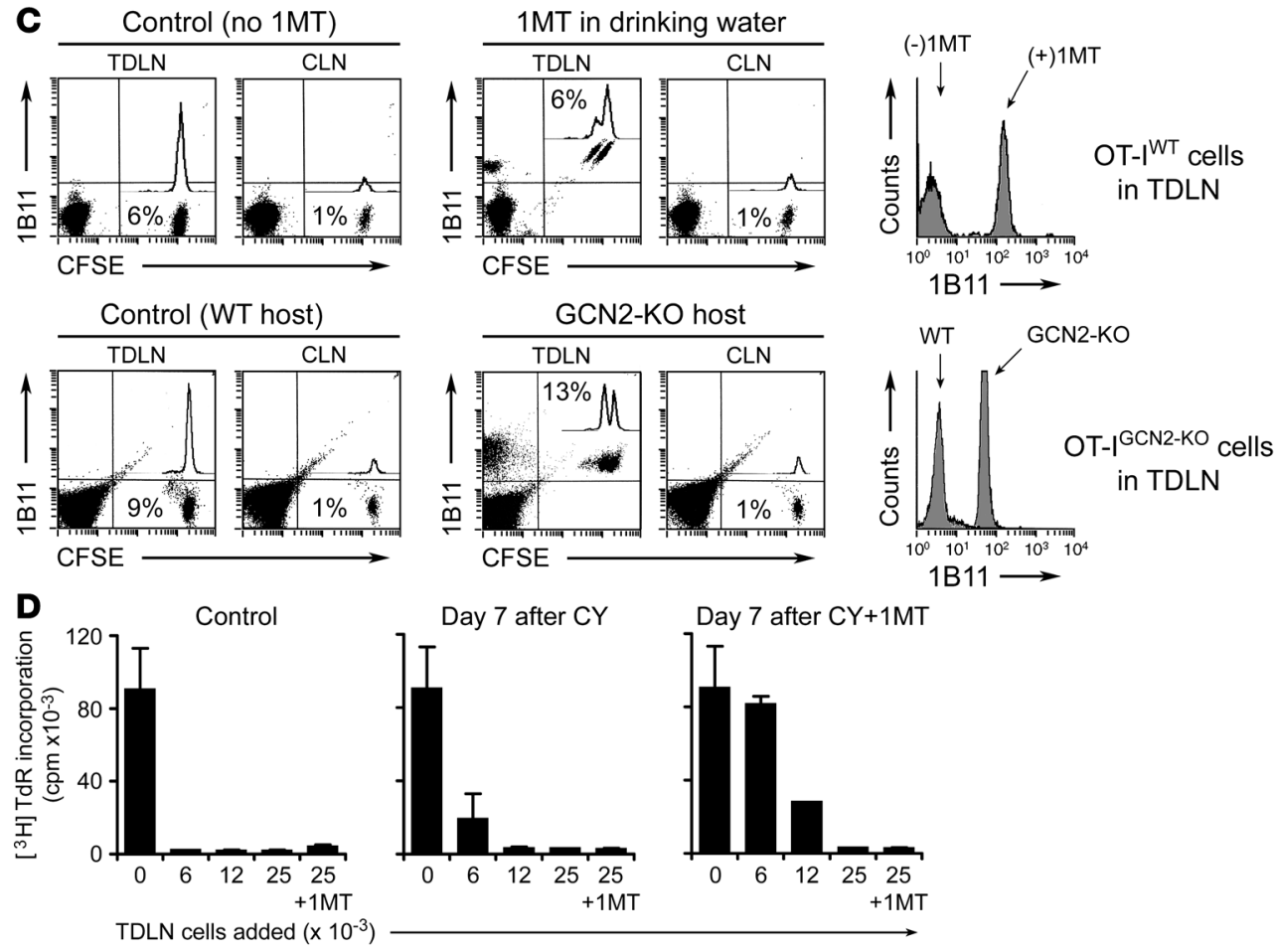

Figure 7

IDO-activated Tregs in TDLNs. (A) Tumors were grown in wild-type or IDO-KO hosts. Tregs from day 7 TDLNs were sorted and added to readout assays (A1 T cells + CBA DCs) with and without PD-1/PD-L blocking antibodies. Mean \pm SD of 4 pooled experiments with B78H1-GM-CSF, 4 experiments with B16-OVA, and 3 experiments with IDO-KO hosts (2 with B78H1-GM-CSF and 1 with B16-OVA). (B) Wild-type mice were treated throughout tumor growth with vehicle control or sustained-release $1 \mathrm{MT}$. Tregs from day 7 tumors were tested in readout assays as described above with added isotype, PD-1/PD-L-blocking antibodies, or a combination of anti-PD-1/PD-L plus IL-2 plus anti-IL-10/TGF- $\beta$ antibodies. One of 3 experiments using B78H1-GM-CSF and B16-OVA. (C) Upper panels: CFSE-labeled OT-I were injected into mice with B16-OVA tumors (days 7-8) with and without oral 1MT administration after transfer. After 4 days, TDLNs and contralateral LNs (CLN) were stained for the 1B11 activation marker. Percentages show the CFSE+ OT-I in total LN cells. Histogram shows $1 \mathrm{~B} 11$ on OT-I in TDLNs. Representative of 4 transfers each. Lower panels: Similar experiments as described above using OT-ןGCN2-KO cells transferred into WT or GCN2-KO hosts bearing B16-OVA tumors. One of 3 similar experiments. (D) B78H1-GM-CSF tumors were treated on day 11 with vehicle (control), cyclophosphamide (CY; $150 \mathrm{mg} / \mathrm{kg}$ ), or cyclophosphamide plus 1MT pellets. Seven days later cells from TDLNs were harvested and added to readout assays (allospecific BM3 T cells plus B6 splenocytes, as described in ref. 1). One group in each readout assay also received 1MT added during the assay, as shown on the last bar of each graph. One of 3 experiments. 


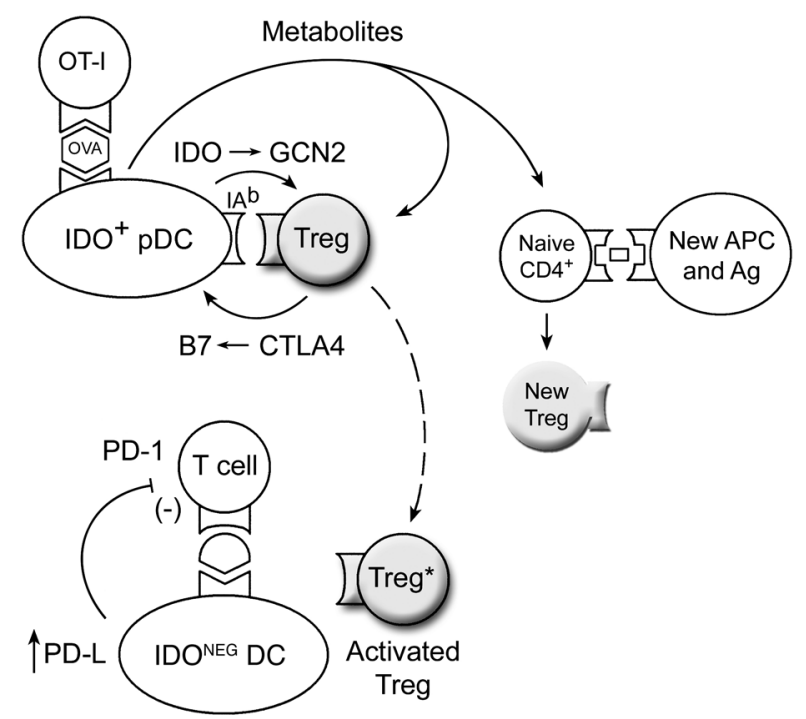

Figure 8

Proposed hypothetical model of IDO-induced Treg activation based on synthesis of results from the in vitro models. The interaction of resting Tregs with IDO+ $\mathrm{pDCs}$ results in activation of the Tregs through a combination of the GCN2 activation and tryptophan metabolites. Activated Tregs then suppress target T cells in an IDO-independent fashion, involving PD-ligand expression on the target DCs, and PD-1 expression (presumably on the target $\mathrm{T}$ cells). In addition, bystander $\mathrm{CD} 4^{+} \mathrm{T}$ cells responding to other antigens, if exposed to the conditions created by activating Tregs and IDO ${ }^{+} \mathrm{pDCs}$, are biased to differentiate into new Tregs.

CTLA4 blockade prevents Treg activation in cocultures. Tregs themselves have been reported to upregulate IDO expression in DCs (27). This occurs via binding of cell surface CTLA4 on Tregs to B7.1/B7.2 molecules on DCs, resulting in B7-mediated induction of IDO $(10,28)$. Consistent with this, we found that the addition of Tregs to cocultures of TDLN pDCs plus OT-I significantly increased IDO enzymatic activity (measured as production of kynurenine, the first major metabolite of tryptophan produced by IDO) and that this Treg-induced enhancement was prevented by blocking CTLA4 in cocultures (Supplemental Figure 5). Likewise, blocking CTLA4 significantly inhibited IDO-induced functional activation of Tregs in cocultures (Figure 5A). Thus, IDO caused activation of Tregs, but a reciprocal interaction with the Tregs appeared necessary for full induction of IDO.

Distinct MHC-restricted and MHC-unrestricted components of activation. We next asked whether interaction with MHC molecules on the pDCs was required for Treg activation. Figure 5B shows that induction of $\mathrm{CHOP}$ expression in Tregs was strictly dependent on interaction with the MHC molecules expressed on the $\mathrm{IDO}^{+} \mathrm{pDCs}$. $\mathrm{CHOP}$ was not induced if the Tregs and $\mathrm{pDC}$ were mismatched at MHC class II (Figure 5B) or if interaction with $\mathrm{MHC}$ was blocked by antibody against IA ${ }^{\mathrm{b}}$ (the MHC-II allele expressed by $\mathrm{B} 6$ mice). Consistent with this, Figure $5 \mathrm{C}$ shows that MHC-mismatched (CBA) Tregs did not become activated during coculture with $\mathrm{IDO}^{+} \mathrm{B} 6 \mathrm{pDCs}$. However, if the CBA Tregs were mixed with MHCmatched (B6 $\left.6^{\text {Thy1.1 }}\right)$ Tregs, then both populations became activated and both mediated suppression via the characteristic IDO-induced PD-1/PD-L-dependent mechanism (Figure 5C). This suggested that an MHC-restricted interaction between the pDCs and Tregs was required to trigger the effects of IDO (perhaps as part of the same CTLA4-dependent activation step described above), but once IDO was triggered it could then affect other Tregs in the cultures in an MHC-unrestricted fashion.

One potential mechanism to explain this MHC-unrestricted effect of IDO might be secretion of soluble metabolites of tryptophan (11). Supplemental Figure 6 presents indirect evidence consistent with this possibility. However, we have not been able to directly reproduce the effects of $\mathrm{IDO}^{+} \mathrm{pDCs}$, including the induction of PD-1/PD-L-mediated suppressor activity, using purified tryptophan metabolites alone. Thus, while the data shown in Supplemental Figure 6 suggest that tryptophan metabolites are important participants in IDO-induced Treg activation, their specific role remains to be determined.

IDO preferentially activates preexisting Tregs. It has been previously shown that IDO can promote de novo differentiation of Foxp $3^{+}$ Tregs from naive $\mathrm{CD}^{+} \mathrm{T}$ cells in vitro (11). We therefore asked whether IDO in our system would induce naive $\mathrm{CD} 4^{+}$cells to differentiate into Foxp $3^{+}$cells. Cocultures were set up as shown in the diagram in Figure 6A and were comprised of $\mathrm{IDO}^{+} \mathrm{pDCs}$, OT-I, feeder cells, mature Tregs (Thy1.1 congenic), and a population of $\mathrm{CD} 4^{+} \mathrm{CD} 25^{-} \mathrm{T}$ cells (naive male-specific A1 T cells isolated from female mice). CBA splenic DCs were also added to serve as APCs for the A1 cells. After 2 days, cocultures were harvested and stained for intracellular Foxp3. Figure $6 \mathrm{~A}$ shows analysis of the $\mathrm{CD} 4+$ population from such an experiment. In the absence of their cognate $\mathrm{H}-\mathrm{Y}$ peptide, none of the A1 cells expressed Foxp3 at the end of culture. In the presence of H-Y peptide, there was upregulation of Foxp3 in up to $95 \%$ of A1 cells, depending on the experiment. Upregulation of Foxp3 was prevented when IDO activity was blocked by 1MT. The smaller dot plots show data from similar assays in which the A1 T cells and OT-I were labeled with CFSE, demonstrating that the A1 T cells remained in a nondivided state when IDO was active but divided when IDO was blocked by $1 \mathrm{MT}$. Further studies demonstrated that the IDO-arrested A1 cells upregulated CD25 and CD44 in response to antigen (thus showing evidence of attempted activation), even though they could not divide (data not shown). Thus, it was the combination of antigenic stimulation by H-Y peptide plus forced cell-cycle arrest by IDO that led to upregulation of Foxp3 in the A1 cells.

To confirm that upregulation of Foxp3 was not a peculiarity of the TCR-transgenic A1 system, similar experiments were performed using nontransgenic (polyclonal) $\mathrm{CD} 4^{+} \mathrm{CD} 25^{-}$cells from wild-type $\mathrm{B} 6$ mice activated with anti-CD3 crosslinking, as in previous studies (11). Identical upregulation of Foxp3 was observed in this system (in which the naive $\mathrm{CD} 4^{+}$cells were identified by CFSE staining; Figure 6B, left dot plot). To ask whether the de novo Foxp3-expressing cells acquired functional activity, the cells were re-sorted and tested for suppressor activity. Figure $6 \mathrm{~B}$ shows that the mature Tregs from these cocultures became potently activated for suppression, whereas the naive $\mathrm{CD}^{+}$population acquired only a small amount of suppressor activity (100-fold less than mature Tregs on a per-cell basis). Thus, within the length of time that our activation assays were performed, newly differentiated Foxp $3^{+}$cells acquired little functional activity in response to IDO, whereas the mature, preexisting Tregs became rapidly and potently activated.

IDO-induced Treg activation in TDLNs. In all the preceding studies, resting Tregs were activated by IDO in vitro. We now asked whether Tregs isolated directly from TDLNs showed evidence of constitutive activation by IDO in vivo. Based on data shown in Figure 3D, findings consistent with IDO-induced Treg activation were defined 
as spontaneous ex vivo suppression that was dependent of the novel PD-1/PD-L pathway and resistant to IL2 and IL-10/TGF- $\beta$ blockade. The "conventional" component of Treg activity was defined as suppression that was reversed by IL2 and IL-10/TGF- $\beta$ blockade and was indifferent to PD-1/PD-L.

Tregs were sorted from TDLNs and added directly to readout assays (A1 T cells + CBA DCs). Figure 7A (left and middle panels) shows that the majority of suppression by TDLN Tregs was prevented by PD-1/PD-L blockade. A small amount of residual PD-1/ $\mathrm{PD}-\mathrm{L}$-independent activity remained at the higher Treg/effector $\mathrm{T}$ cell ratios, consistent with a mixture of both conventional and IDOinduced forms of suppression. Based on the shift in IC50, 75\%-90\% of suppression by TDLN Tregs appeared mediated via the PD-1/ $\mathrm{PD}-\mathrm{L}$ pathway. In contrast, when tumors were grown in IDO-KO mice, the Tregs in TDLNs completely lacked the PD-1/PD-L-mediated component of suppression (Figure 7A, right panel).

Similar results were obtained when the IDO pathway was pharmacologically inhibited by administering $1 \mathrm{MT}$ during the period of tumor growth (Figure 7B). To test the hypothesis that the residual, non-PD-1/PD-L-mediated component of suppression represented conventional Treg activity, we asked whether exogenous IL-2 plus anti-IL-10/TGF- $\beta$ blockade would reverse this residual component of Treg suppression. Figure 7B shows that this manipulation completely reversed all of the remaining components of suppression. In the case of tumors grown in the absence of IDO (mice administered 1MT; Figure 7B), the conventional (IL-2/anti-IL-10/TGF- $\beta$-reversible) form of suppression accounted for all of the Treg activity in TDLNs, and none of the Treg activity was PD-1/PD-L dependent.

Inbibition of $T$ cell responses in TDLNs in vivo. We next asked whether in vivo $\mathrm{T}$ cell responses were suppressed in TDLNs. OT-1 were labeled with CFSE tracking dye and injected intravenously into mice bearing established B16-OVA tumors (which express the cognate antigen for OT-I). Figure 7C (upper panels) shows that OT-I preferentially accumulated in the TDLN 4 days after injection ( $6 \%$ of cells in TDLN versus $1 \%$ in contralateral LN), but they showed no cell division and no evidence of activation (assessed as upregulation of the $1 \mathrm{~B} 11 \mathrm{~T}$ cell activation marker; ref. 29). To ask whether this lack of response was related to IDO expression, recipient mice were treated with $1 \mathrm{MT}$. When IDO was blocked with 1MT, OT-I in TDLNs became able to uniformly upregulate the activation marker 1B11 (Figure 7C, histograms), thus showing evidence of attempted activation, although they were still not able to undergo extensive cell division.

The use of 1MT could not distinguish between direct suppression of OT-I by IDO itself and an IDO-induced activation of host suppressor cells (e.g., IDO-activated Tregs). To eliminate any direct effect of IDO on OT-I, we used OT-I mice bred onto the GCN2-KO background (OT-IGCN2-KO mice). This rendered the OT-IGCN2-KO $\mathrm{T}$ cells refractory to the direct suppressive effects of IDO, as we have previously shown (9), but they remained fully susceptible to suppression by Tregs, since Treg-mediated suppression was independent of IDO (cf. Figure 3B). Since IDO could not directly suppress the OT-IGCN2-KO cells, any effect of IDO would have to be exerted via IDO-responsive host suppressor cells. This host response to IDO could be controlled by making the recipient mice either GCN2 sufficient or GCN2-KO, as shown in Figure 7C (lower panels). When recipient mice were GCN2 sufficient, transferred OT-IGCN2-KO cells remained fully suppressed in TDLNs; however, the same OT-IGCN2-KO cells became able to activate if the recipient mice were GCN2-KO (and hence unable to respond to IDO). These data thus supported the preceding data using 1MT and, taken together, were consistent with a population of IDOresponsive suppressor cells in TDLNs, derived from the host and activated by IDO in a GCN2-dependent fashion.

Chemotherapy plus $1 M T$ in vivo depletes suppressor activity in TDLNs. From a clinical standpoint, constitutive activation of Tregs in TDLNs could represent a formidable barrier to immunotherapy. Certain chemotherapeutic drugs such as cyclophosphamide have been reported to partially reduce the number and/or function of Tregs $(30,31)$. We and others have shown that $1 \mathrm{MT}$ displays immune-mediated synergistic antitumor effects when combined with chemotherapy $(6,7)$. Therefore, we asked whether cyclophosphamide combined with $1 \mathrm{MT}$ could reduce the suppressor activity found in TDLNs. For these experiments, we measured the suppressor activity in total, unfractionated TDLN cells, as previously described (1), because the cell number in TDLNs after chemotherapy was too small to permit sorting of individual cell populations. Figure 7D shows that in untreated control mice, the TDLN cells were intensely suppressive in the readout assays (proliferation of $\mathrm{CD}^{+} \mathrm{T}$ cells). This suppression was not affected by the addition of $1 \mathrm{MT}$ to the readout assays (final bar in each graph), consistent with a significant component of suppression by activated Tregs. Treatment with cyclophosphamide alone reduced the suppressive activity only slightly. However, administration of cyclophosphamide followed by $1 \mathrm{MT}$ significantly reduced the suppressor activity by TDLN cells (Figure 7D). This suggested that the potently suppressive milieu in TDLNs could be partially alleviated by the combination of chemotherapy plus $1 \mathrm{MT}$.

\section{Discussion}

The current study demonstrates for the first time a mechanistic link between IDO, functional activation of Tregs, and the PD-1/ PD-L pathway. Each of these mechanisms is known to be independently important in tumor immunology, and strategies targeting each mechanism are currently in clinical trials or in active preclinical development. We now show that these 3 powerful regulatory mechanisms are tightly linked at the level of Treg activation in the TDLN. This linked pathway constitutes a major contributor to the intensely immunosuppressive milieu present in TDLNs. Since this suppressive milieu drives $T$ cell anergy and unresponsiveness to tumor antigens presented in the TDLNs (32), identification of molecular mechanisms contributing to this suppression represents an important goal in cancer immunotherapy.

Our findings suggest a hypothetical model (Figure 8) in which IDO-induced Treg activation proceeds via a self-amplifying loop. We hypothesize that when $\mathrm{IDO}^{+} \mathrm{pDCs}$ present antigen to effector $\mathrm{T}$ cells in the presence of mature, resting Tregs, this initiates a GCN2-dependent activation of the Tregs by IDO. In other cells, GCN2 is known to activate a downstream stress-response pathway, resulting in a coordinated program of changes in gene expression $(26,33)$. In the case of $\mathrm{CD}^{+}$effector $\mathrm{T}$ cells, we have shown that activation of the GCN2 pathway leads to cell cycle arrest and anergy (9). In the case of Tregs, we now show that GCN2 signaling is critical for allowing IDO-induced functional activation. Based on our data (Figure 5A and Supplemental Figure 5), we speculate that the activating Tregs reciprocally induce high levels of IDO in pDCs, via CTLA4-B7 interaction (27), leading to increased production of tryptophan metabolites. These metabolites then complete the full activation of the Tregs (as suggested by data shown in 
Figure 5C and Supplemental Figure 6), resulting in emergence of the novel, highly potent PD-1/PD-L-dependent form of suppression that we describe. This model is speculative, but it is consistent with our data, and the 2 key features of the model are clear and well supported: direct IDO-induced activation of mature Tregs and PD-1/PD-L-dependent suppression by IDO-activated Tregs.

A role for PD-1/PD-L as a downstream suppressor mechanism for Tregs has not been previously described. However, induction of a another suppressive B7 family member, B7-H4, on APCs by Tregs has been recently shown (25). In our system, the PD-1/PD-L mechanism of suppression was only found with the IDO-induced form of Treg activation and was not seen with the widely studied anti-CD3-induced form of Treg activation. The PD-1/PD-L pathway has been the focus of considerable interest because it has been found to mediate clonal exhaustion and $\mathrm{T}$ cell anergy in HIV and other chronic viral infections (34) as well as tolerance to self antigens and immune suppression in cancer (35). Our findings provide a novel mechanistic link between the PD-1/PD-L system, Tregs, and IDO.

Tregs isolated from TDLNs in vivo were constitutively activated, displaying spontaneous suppressor activity that was as potent as the highest levels reported for Tregs extensively activated in vitro $(15,16)$. The majority of this constitutive Treg activity in TDLNs was mediated via the novel IDO-induced, PD-1/PD-L-dependent mechanism. We demonstrate the existence of 2 distinct, clearly distinguishable forms of Treg activity - the conventional form elicited by anti-CD3 crosslinking, in which suppression was dependent on IL-10/TGF- $\beta$, was reversed by IL- 2 , and was unaffected by PD-1/PD-L blockade; and the novel IDO-induced form, which was not dependent on IL-10/TGF- $\beta$, was not reversed by IL-2, and was strictly dependent on the PD-1/PD-L pathway. Under IDO-sufficient conditions, $75 \%-90 \%$ of the constitutive Treg activity in TDLNs was due to the IDO-induced form of Treg activity. This IDO-induced component was completely lost when tumors were grown in IDO-KO mice or in mice treated with an IDO-inhibitor drug during tumor growth. Under these chronically IDO-deficient conditions, tumors showed a compensatory increase in the form of Treg activity that was not dependent on IDO, consistent with emergence of tumor escape variants (36). However, while tumors were thus able to compensate for artificial genetic or pharmacologic ablation of IDO, from a clinical standpoint human patients would normally be IDO sufficient. Thus, the key observation in our system was that $75 \%-90 \%$ of the naturally occurring Treg activity in TDLNs was of the IDO-induced, PD-1/PD-L-dependent form.

In vitro, IDO activity also promoted de novo upregulation of Foxp3 expression in naive $\mathrm{CD} 4^{+} \mathrm{T}$ cells. This finding is not novel, since the pathway has already been described by Fallarino and colleagues (11). In our system, the mature, preexisting Tregs activated by IDO were 100 -fold more potent on a per-cell basis than the newly differentiated Foxp $3^{+}$cells. In human T cells, it is known that Foxp3 upregulation does not necessarily connote stable commitment to Treg differentiation $(37,38)$, so it is possible that not all of the newly derived Foxp $3^{+}$cells would go on to become Tregs. Nevertheless, it is relevant to note that IDO is potentially linked to the Treg lineage at 2 points, the rapid and potent activation of mature Tregs that we describe and the potential for de novo differentiation of new Tregs.

IDO-induced Treg activation was almost entirely prevented by blockade of CTLA4. CTLA4 has multiple regulatory roles in the immune system, most of which are intrinsic to the CTLA4 ${ }^{+} \mathrm{T}$ cells themselves; however, it is also known that CTLA4 can induce
IDO expression in DCs, via back-signaling through B7 molecules (27). We hypothesize that CTLA4 on Tregs delivers a signal to $\mathrm{IDO}^{+} \mathrm{pDCs}$ that enhances their normal level of IDO enzymatic activity and thus increases the production of immunoregulatory metabolites. Interpretation of such studies is complex because it is difficult to separate cell-autonomous effects of the antibody on Treg function from the effect of the antibody on IDO, so further studies are required. However, from a therapeutic standpoint, anti-CTLA4 antibodies are in late-stage clinical trials (39), so it is of interest to note that CTLA4 blockade also interrupts the novel IDO/Treg/PD-L pathway.

The human counterpart of the IDO+ $\mathrm{pDCs}^{+}$in mouse TDLNs is not yet established, and human and mouse DC subsets do not always correspond. However, a prominent population of IDO-expressing cells is observed in many human TDLNs (40), displaying a characteristic plasmacytoid morphology (41). Recently, human plasmacytoid DCs $\left(\mathrm{CD} 123^{+} \mathrm{BDCA}^{+}\right)$have been shown to upregulate IDO in response to HIV infection (42); thus, authentic human pDCs can be induced to express IDO. Further investigation will be required to specifically identify the IDO-expressing cells found in human TDLNs. Future studies will also be needed to address the possible developmental roles of IDO and GCN2 in the differentiation of the Treg lineage. Preliminary studies demonstrate selective but significant functional defects in Tregs derived from IDO-KO, GCN2-KO, and CHOP-KO mice, suggesting that the IDO pathway may have broader importance for aspects of normal Treg differentiation.

The current study suggests that patients with cancer may have abnormally increased Treg activity in TDLNs, due in part to the effects of IDO. Once tumors are established, simply blocking IDO was not sufficient to fully reverse the suppressive milieu in the TDLN (Figure 7C). But even in established tumors, blocking IDO allowed initial activation of tumor-specific effector T cells in TDLNs, with attempted cell division. Combining IDO-inhibitor drugs with chemotherapy may further help to reverse the established suppressive milieu in TDLNs. Therapeutic strategies to block IDO, tumorinduced Tregs, and the PD-1/PD-L pathway are all currently in clinical or preclinical development. Our demonstration of a molecular link uniting all 3 of these potent immunosuppressive mechanisms may have significant implications for cancer immunotherapy.

\section{Methods}

Additional details of Methods are given in Supplemental Methods online. Mice and tumors. Animal studies were approved by the Institutional Animal Care and Use Committee of the Medical College of Georgia. TCR-transgenic OT-I mice (CD8 ${ }^{+}, \mathrm{B} 6$ background, recognizing the SIINFEKL peptide of ovalbumin on $\mathrm{H}_{2} \mathrm{~K}^{\mathrm{b}}$; ref. 43) and B6.PL-Thy1a/CyJ mice (congenic for the $\mathrm{B} 6$ background but bearing the Thy1.1 allele) were purchased from The Jackson Laboratory. GCN2-KO mice (B6 background) were a generous gift from the laboratory of David Ron (New York University School of Medicine, New York, New York, USA) and have been previously described (9). A1 mice (CBA background, recognizing an H-Y peptide presented on $\mathrm{IE}^{\mathrm{k}}$ ) (44), BM3 mice (CBA background, recognizing $\mathrm{H} 2 \mathrm{~K}^{\mathrm{b}}$ as an alloantigen; ref. 45), and IDO-KO mice (B6 and CBA backgrounds; refs. 10, 46) have been previously described.

Tumor implantation is described in detail in Supplemental Methods. Cell lines used were B78H1-GM-CSF (a subline of B16 transfected with GM-CSF [ref. 14], as used in our previous studies of IDO ${ }^{+}$pDCs [refs. 1, 9]); the B16F10 subline of B16 (ATCC) and B16-OVA subline (parental B16F10 transfected with full-length OVA, clone MO4 [ref. 47], a gift from Alan Houghton (Memorial Sloan-Kettering Cancer Center, New York, New York, 
USA). The use of OVA as a model tumor antigen was informative in our system, since the goal was to detect the suppression of immune response to tumor antigens; thus, a strong nominal antigen was an advantage. IDO ${ }^{+}$ pDCs and activated Tregs found in TDLNs of all 3 tumor lines were similar. As in our previous publications $(1,9)$, most experiments requiring sorted pDCs used the B78H1-GM-CSF tumors because these gave the highest yield of pDCs (see Supplemental Figure 1). However, pDCs from tumors without GM-CSF gave similar functional results, and all key findings were confirmed with tumors with and without GM-CSF.

Reagents and 1MT. 1MT (catalog no. 45,248-3; Sigma-Aldrich) was prepared as described (9) and used at a final concentration of $200 \mu \mathrm{M}$. Delivery of $1 \mathrm{MT}$ by sustained-release subcutaneous pellets $(5 \mathrm{mg} /$ day $)$ was as previously described (7). For oral delivery, 1MT was added to drinking water at $2 \mathrm{mg} / \mathrm{ml}$. Recombinant mouse IL-2 (R\&D Systems) was used at $10 \mathrm{ng} / \mathrm{ml}$. Blocking antibodies against PD-L1/B7-DC (clone MIH7) (48), PD-L2 (TY25) (49), and PD-1 (J43) (50) were used as a cocktail at $50 \mu \mathrm{g} / \mathrm{ml}$ each (or rat IgG1 isotype control). Anti-CTLA4 antibody (clone 9H10, used at $10 \mu \mathrm{g} / \mathrm{ml}$ ) and rat anti-IL-10 receptor antibody (used at $100 \mu \mathrm{g} / \mathrm{ml}$, clone 1B1.3a) were from BD Biosciences; anti-mouse I-A ${ }^{b}$ (used at $100 \mu \mathrm{g} / \mathrm{ml}$ ) and IgM isotype control were from Southern Biotech; chicken anti-TGF- $\beta$ / $\beta 2 / \beta 3$ (MAB1835, used at $100 \mu \mathrm{g} / \mathrm{ml}$ ) was from R\&D Systems.

Ex vivo Treg assays. Tregs $\left(\mathrm{CD} 4^{+} \mathrm{CD} 25^{+}\right)$were sorted from $2-4$ pooled TDLNs and added directly to readout assays containing $1 \times 10^{5} \mathrm{CD}^{+}$ A1 cells, $2 \times 10^{3} \mathrm{CD} 11 \mathrm{c}^{+}$DCs from CBA spleen, and $100 \mathrm{nM} \mathrm{H}-\mathrm{Y}$ peptide (REEALHQFRSGRKPI). All cultures were performed in V-bottom wells. For both Tregs and pDCs, it was important to perform sorts rapidly, collect cells in complete medium on ice, and transfer them promptly into culture, in order to preserve viability and function.

Treg activation cultures and readout assays. Sorting of $\mathrm{pDCs}$ from TDLNs was performed as described $(1,9)$. The $\mathrm{pDC}$ fraction $\left(\mathrm{CD} 11 \mathrm{c}^{+} \mathrm{B} 220^{+}\right)$was sorted from 2-6 pooled TDLNs (days 7-11 of tumor growth) and collected in medium on ice. Activation cultures contained $2 \times 10^{3} \mathrm{pDCs}, 1 \times 10^{5}$ sorted $\mathrm{CD}^{+}$OT-I, $100 \mathrm{nM}$ SIINFEKL peptide, and $5 \times 10^{3}$ sorted $\mathrm{CD} 4^{+} \mathrm{CD} 25^{+}$ Tregs from spleens of $\mathrm{B} 6$ mice without tumors. All cultures received a feeder layer of $1 \times 10^{5} \mathrm{~T}$ cell-depleted spleen cells $\left(\mathrm{CD}^{-}{ }^{-} \mathrm{CD}^{-}\right)$) as described in Supplemental Methods. For anti-CD3-induced activation, the same cultures received $200 \mu \mathrm{M} 1 \mathrm{MT}$ to block IDO plus $0.1 \mu \mathrm{g} / \mathrm{ml}$ anti-CD3 $\mathrm{mAb}$ (clone 145-2C11; BD Biosciences - Pharmingen) and $10 \mathrm{ng} / \mathrm{ml} \mathrm{IL-2}$. We routinely added IL-2 to the anti-CD3 activation cultures, although this did not have any further enhancing effect on suppressor activity over anti-CD3 alone, presumably because adequate IL-2 was contributed by the activating OT-I
(51). After 2 days, cultures were harvested and stained for CD4 and Tregs were isolated by sorting for $\mathrm{CD}^{+}$cells. Preliminary studies showed that sorting on either total $\mathrm{CD} 4{ }^{+}$Tregs or the $\mathrm{CD} 4{ }^{+} \mathrm{CD} 62 \mathrm{~L}^{\text {hi }}$ subset of Tregs gave equivalent results, so the total $\mathrm{CD} 4^{+}$Treg population was routinely used. Re-sorted Tregs were added to readout assays containing $1 \times 10^{5} \mathrm{~A} 1$ cells,

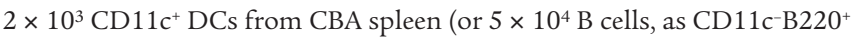
spleen cells), and H-Y peptide. For assays performed in transwells, Multiwell 96-well insert plates ( $1 \mu \mathrm{M}$ pore size; Falcon; $\mathrm{BD})$ were used and the number of cells in all groups was doubled.

$D C$ and $T$ cell adoptive transfer. The DC adoptive transfer model and adoptive transfer of CFSE-labeled T cells have been previously described $(1,9)$, and detailed methods are given in Supplemental Methods.

Statistics. Individual thymidine incorporation assays were performed in triplicate or quadruplicate wells for each data point, and for these analyses, error bars in the figures indicate SD of replicate wells. Multiple treatment groups in each experiment were compared using ANOVA. In cases where 1 representative experiment of several is shown, each independent replicate experiment showed comparable statistical significance between the same groups by ANOVA. Where multiple experiments were combined for analysis, raw thymidine incorporation counts were normalized to the control (wells without Tregs) in each experiment to permit comparison across multiple experiments. For these analyses, the error bars in the figures indicate the SD of the pooled data.

\section{Acknowledgments}

The authors thank Makio Iwashima and Pandelakis Koni for insightful discussion; Jingping Sun, Joyce Wilson, Judy Gregory, and Doris McCool for expert technical assistance; and Jeanene Pihkala for expert cell sorting. D.H. Munn and A.L. Mellor have intellectual property interests in the therapeutic use of IDO and IDO inhibitors, and receive consulting income from NewLink Genetics Inc. This work was supported by NIH grants CA103320, CA096651, and CA112431 (to D.H. Munn) and HD41187 and AI063402 (to A.L. Mellor).

Received for publication February 21, 2007, and accepted in revised form May 29, 2007.

Address correspondence to: David H. Munn, Room CN-4141, Medical College of Georgia, Augusta, Georgia 30912, USA. Phone: (706) 721-7141; Fax: (706) 721-8732; E-mail: dmunn@mail.mcg.edu.
1. Munn, D.H., et al. 2004. Expression of indoleamine 2,3-dioxygenase by plasmacytoid dendritic cells in tumor-draining lymph nodes. J. Clin. Invest. 114:280-290. doi:10.1172/JCI200421583.

2. Munn, D.H., et al. 1998. Prevention of allogeneic fetal rejection by tryptophan catabolism. Science. 281:1191-1193.

3. Gurtner, G.J., et al. 2003. Inhibition of indoleamine 2,3-dioxygenase augments trinitrobenzene sulfonic acid colitis in mice. Gastroenterology. 125:1762-1773.

4. Liu, H., Liu, L., Fletcher, B.S., and Visner, G.A. 2006. Sleeping Beauty-based gene therapy with indoleamine 2,3-dioxygenase inhibits lung allograft fibrosis. FASEB J. 20:2384-2386.

5. Uyttenhove, C., et al. 2003. Evidence for a tumoral immune resistance mechanism based on tryptophan degradation by indoleamine 2,3-dioxygenase. Nat. Med. 9:1269-1274.

6. Muller, A.J., Duhadaway, J.B., Donover, P.S., Sutanto-Ward, E., and Prendergast, G.C. 2005. Inhibition of indoleamine 2,3-dioxygenase, an immunoregulatory target of the cancer suppression gene Bin1, potentiates cancer chemotherapy. Nat. Med. 11:312-319.
7. Hou, D.Y., et al. 2007. Inhibition of IDO in dendritic cells by stereoisomers of 1-methyl-tryptophan correlates with anti-tumor responses. Cancer Res. 67:792-801.

8. Munn, D.H., and Mellor, A.L. 2007. Indoleamine 2,3-dioxygenase and tumor-induced tolerance. J. Clin. Invest. 117:1147-1154. doi:10.1172/JCI31178.

9. Munn, D.H., et al. 2005. GCN2 kinase in T cells mediates proliferative arrest and anergy induction in response to indoleamine 2,3-dioxygenase. Immunity. 22:633-642.

10. Mellor, A.L., et al. 2003. Cutting edge: induced indoleamine 2,3 dioxygenase expression in dendritic cell subsets suppresses $\mathrm{T}$ cell clonal expansion. J. Immunol. 171:1652-1655.

11. Fallarino, F., et al. 2006. The combined effects of tryptophan starvation and tryptophan catabolites down-regulate $T$ cell receptor zeta-chain and induce a regulatory phenotype in naive $\mathrm{T}$ cells. J. Immunol. 176:6752-6761.

12. Zou, W. 2006. Regulatory T cells, tumour immunity and immunotherapy. Nat. Rev. Immunol. 6:295-307.

13. Munn, D.H., et al. 1999. Inhibition of T cell proliferation by macrophage tryptophan catabolism.
J. Exp. Med. 189:1363-1372.

14. Huang, A.Y., et al. 1994. Role of bone marrowderived cells in presenting MHC class I-restricted tumor antigens. Science. 264:961-965.

15. McHugh, R.S., et al. 2002. CD4(+)CD25(+) immunoregulatory $\mathrm{T}$ cells: gene expression analysis reveals a functional role for the glucocorticoidinduced TNF receptor. Immunity. 16:311-323.

16. Caramalho, I., et al. 2003. Regulatory T cells selectively express Toll-like receptors and are activated by lipopolysaccharide. J. Exp. Med. 197:403-411.

17. Thornton, A.M., and Shevach, E.M. 1998. CD4+CD25+ immunoregulatory $\mathrm{T}$ cells suppress polyclonal $\mathrm{T}$ cell activation in vitro by inhibiting interleukin 2 production. J. Exp. Med. 188:287-296.

18. Nishikawa, H., et al. 2005. Definition of target antigens for naturally occurring CD4(+) CD25(+) regulatory T cells. J. Exp. Med. 201:681-686.

19. Thornton, A.M., and Shevach, E.M. 2000. Suppressor effector function of CD4+CD25+ immunoregulatory $\mathrm{T}$ cells is antigen nonspecific. J. Immunol. 164:183-190.

20. Munn, D.H., Sharma, M.D., and Mellor, A.L. 2004. Ligation of B7-1/B7-2 by human CD4+ T cells trig- 
gers indoleamine 2,3-dioxygenase activity in dendritic cells. I. Immunol. 172:4100-4110.

21. Thornton, A.M., Piccirillo, C.A., and Shevach, E.M 2004. Activation requirements for the induction of CD4+CD25+ T cell suppressor function. Eur. J. Immunol. 34:366-376

22. Bluestone, J.A., and Tang, Q. 2005. How do CD4+ $\mathrm{CD} 25+$ regulatory $\mathrm{T}$ cells control autoimmunity? Curr. Opin. Immunol. 17:638-642.

23. Probst, H.C., McCoy, K., Okazaki, T., Honjo, T. and van den Broek, M. 2005. Resting dendritic cells induce peripheral CD8+ T cell tolerance through PD-1 and CTLA-4. Nat. Immunol. 6:280-286.

24. Curiel, T.J., et al. 2003. Blockade of B7-H1 improves myeloid dendritic cell-mediated antitumor immunity. Nat. Med. 9:562-567.

25. Kryczek, I., et al. 2006. Cutting edge: induction of B7-H4 on APCs through IL-10: novel suppressive mode for regulatory T cells. J. Immunol. 177:40-44.

26. Harding, H.P., et al. 2003. An integrated stress response regulates amino acid metabolism and resistance to oxidative stress. Mol. Cell. 11:619-633.

27. Fallarino, F., et al. 2003. Modulation of tryptophan catabolism by regulatory $\mathrm{T}$ cells. Nat. Immunol. 4:1206-1212.

28. Grohmann, U., et al. 2002. CTLA-4-Ig regulates tryptophan catabolism in vivo. Nat. Immunol. 3:1097-1101

29. Harrington, L.E., Galvan, M., Baum, L.G., Altman, J.D., and Ahmed, R. 2000. Differentiating between memory and effector CD8 T cells by altered expression of cell surface O-glycans. J. Exp. Med. 191:1241-1246.

30. Ghiringhelli, F., et al. 2004. CD4+CD25+ regulatory $T$ cells suppress tumor immunity but are sensitive to cyclophosphamide which allows immunotherapy of established tumors to be curative. Eur. J. Immunol. 34:336-344.
31. Lutsiak, M.E., et al. 2005. Inhibition of CD4+25+ $\mathrm{T}$ regulatory cell function implicated in enhanced immune response by low-dose cyclophosphamide. Blood. 105:2862-2868.

32. Munn, D.H., and Mellor, A.L. 2006. The tumordraining lymph node as an immune-privileged site. Immunol. Rev. 213:146-158.

33. Wek, R.C., Jiang, H.Y., and Anthony, T.G. 2006 Coping with stress: eIF2 kinases and translational control. Biochem. Soc. Trans. 34:7-11.

34. Sharpe, A.H., Wherry, E.J., Ahmed, R., and Freeman, G.J. 2007. The function of programmed cell death 1 and its ligands in regulating autoimmunity and infection. Nat. Immunol. 8:239-245.

35. Okazaki, T., and Honjo, T. 2006. The PD-1-PD-L pathwayin immunological tolerance. Trends Immunol. 27:195-201.

36. Zitvogel, L., Tesniere, A., and Kroemer, G. 2006. Cancer despite immunosurveillance: immunoselection and immunosubversion. Nat. Rev. Immunol. 6:715-727.

37. Wang, J., Ioan-Facsinay, A., van der Voort, E.I., Huizinga, T.W., and Toes, R.E. 2007. Transient expression of FOXP3 in human activated nonregulatory CD4+ T cells. Eur. J. Immunol. 37:129-138.

38. Gavin, M.A., et al. 2006. Single-cell analysis of normal and FOXP3-mutant human T cells: FOXP3 expression without regulatory $\mathrm{T}$ cell development. Proc. Natl. Acad. Sci. U. S. A. 103:6659-6664.

39. Peggs, K.S., Quezada, S.A., Korman, A.J., and Allison, J.P. 2006. Principles and use of anti-CTLA4 antibody in human cancer immunotherapy. Curr. Opin. Immunol. 18:206-213.

40. Munn, D.H., et al. 2002. Potential regulatory function of human dendritic cells expressing indoleamine 2,3-dioxygenase. Science. 297:1867-1870.

41. Lee, J.R., et al. 2003. Pattern of recruitment of immunoregulatory antigen presenting cells in malignant melanoma. Lab. Invest. 83:1457-1466.

42. Boasso, A., et al. 2007. HIV inhibits CD4+ T-cell proliferation by inducing indoleamine 2,3-dioxygenase in plasmacytoid dendritic cells. Blood. 109:3351-3359.

43. Hogquist, K.A., et al. 1994. T cell receptor antagonist peptides induce positive selection. Cell. 76:17-27.

44. Zelenika, D., et al. 1998. Rejection of H-Y disparate skin grafts by monospecific CD4+ Th1 and Th2 cells: no requirement for CD8+ $\mathrm{T}$ cells or $\mathrm{B}$ cells. J. Immunol. 161:1868-1874.

45. Tarazona, R., et al. 1996. Effects of different antigenic microenvironments on the course of $\mathrm{CD}^{+} \mathrm{T}$ cell responses in vivo. Int. Immunol. 8:351-358.

46. Baban, B., et al. 2005. A minor population of splenic dendritic cells expressing CD19 mediates IDO-dependent $\mathrm{T}$ cell suppression via type 1 interferon-signaling following B7 ligation. Int. Immunol. 17:909-919.

47. Falo, L.D., Jr., Kovacsovics-Bankowski, M., Thompson, K., and Rock, K.L. 1995. Targeting antigen into the phagocytic pathway in vivo induces protective tumour immunity. Nat. Med. 1:649-653.

48. Tsushima, F., et al. 2003. Preferential contribution of B7-H1 to programmed death-1-mediated regulation of hapten-specific allergic inflammatory responses. Eur. J. Immunol. 33:2773-2782.

49. Yamazaki, T., et al. 2002. Expression of programmed death 1 ligands by murine $\mathrm{T}$ cells and APC. J. Immunol. 169:5538-5545.

50. Agata, Y., et al. 1996. Expression of the PD-1 antigen on the surface of stimulated mouse $\mathrm{T}$ and $\mathrm{B}$ lymphocytes. Int. Immunol. 8:765-772.

51. Thornton, A.M., Donovan, E.E., Piccirillo, C.A., and Shevach, E.M. 2004. Cutting edge: IL-2 is critically required for the in vitro activation of CD4+CD25+ T cell suppressor function. J. Immunol. 172:6519-6523 